\title{
Gastric cancer treated in 1991 in Japan: data analysis of nationwide registry
}

\author{
Japanese Gastric Cancer Association Registration Committee: Keitchi Maruyama, Michio Kaminishi, \\ Ken-ichi Hayashi, Yoh Isobe, Ichiro Honda, Hitoshi Katai, Kuniyoshi Arai, Yasuhiro Kodera, \\ and Atsushi Nashimoto \\ Data Center of the Japanese Gastric Cancer Association, c/o Department of Surgical Oncology, National Cancer Center Hospital, \\ 5-1-1 Tsukiji, Chuo-ku, Tokyo 104-0045, Japan
}

\begin{abstract}
The Japanese Gastric Cancer Association Registration Committee reported the treatment results and causes of death of patients with primary gastric cancer treated in 1991 at the leading hospitals in Japan. Data of 8851 patients with primary gastric cancer were collected from 113 hospitals, and data of 7935 patients with gastric resection were finally analyzed. The lost-to-follow-up rate was $6.9 \%$; the direct death rate was $1.0 \%$. The cumulative 5-year survival rate (5YSR) of all the patients was $68.2 \%$; $89.9 \%$ for Stage I, $69.1 \%$ for Stage II, $\mathbf{4 3 . 5} \%$ for Stage III, and $\mathbf{9 . 9} \%$ for Stage IV. Characteristic findings of the analyzed data were (1) high proportion of early-stage cancer, (2) high resection rate, (3) low mortality rate, (4) low incidence of upper-third cancer, (5) poor treatment results in cases with scirrhous cancer, infiltrating growth, and marked lymphatic or venous invasion, and (6) predominance of systematic (D2) and extended lymphadenectomies possibly resulting in reducing local recurrence and improving survivals.
\end{abstract}

Key words Gastric cancer · Nationwide registry · Japan · Treatment results

\section{Introduction}

In 1963, a nationwide registry of gastric cancer patients was launched by the Japanese Research Society for Gastric Cancer. The society was reorganized into the Japanese Gastric Cancer Association (JGCA) in 1997, but the data collection has been continued by the new association. Requirements to participate in the activity were strict: leading national or regional hospitals having full-time specialists of diagnosis, surgery, and pathology, and $90 \%$ and more follow-up rate. In the period 1963-1990, data were collected and analyzed. The re-

Offprint requests to: K. Maruyama

Received: February 3, 2005 / Accepted: February 14, 2006 sults were published in the annual and special reports; total 54 volumes [1].

The registry system was changed from paper form to a personal computer data-collection system in 1991. The first data including cumulative 5-year survivals were collected in 1998. Data of 8851 patients were registered from 113 hospitals, and 543 were excluded because of the final diagnosis as a recurrence in the gastric remnants, histological diagnosis of gastric sarcoma, or lack of essential data. Data of 8308 patients with primary gastric carcinoma were finally analyzed in this report. The JGCA Registration Committee intends to wake known the 1991 treatment results at the leading hospitals in Japan.

\section{Member hospitals}

Data of gastric cancer patients were collected from the following 113 surgical departments (in alphabetical order).

Aichi Cancer Center, Aichi Prefectural Hosp, Akita Univ Dept 2, Cancer Institute, Center for Adult Diseases Osaka, Chiba Cancer Center, Chiba Univ Dept 2, Defense Med Coll Dept 1, Dokkyo Med Coll Dept 2, Fujita Health Univ Hosp 2, Fujita Health Univ Funabiki-Dept, Fujita Health Univ MiuraDept, Fukui Med Coll Dept 1, Fukui Red Cross Hosp, Fukui Saiseikai Hosp, Fukuoka City Hosp, Fukuoka Univ Chikushi Hosp, Fukushima Med Coll Dept 1, Gifu Univ Dept 2, Gunma Univ Dept 2, Hamamatsu Univ Dept 2, Himeji Chuo Hosp, Hirosaki Univ Dept 1, Hiroshima City Hosp, Hiroshima Univ Dept 2, Hyogo Cancer Center, Ishikawa Chuo Hosp, Iwate Fukuoka Hosp, Iwate Med Coll Dept 1, International Med Center Tokyo, Kagawa Med Coll Dept 1, Kagoshima Nanpu Hosp, Kagoshima Univ Dept 1, Kanagawa Cancer Center, Kansai Med Coll Dept 2, Karasawa Hosp, Kawasaki Ida Hosp, Keio Univ Dept of Surg, Kinki Chuo Hosp, Kinki Univ Dept 1, Kita-kyushu Med Center, Kitasato Univ East Hosp, Kobe Univ Dept 1, Kumamoto Univ Dept 2, Kurume Univ Dept of 
Surg, Kyoto Prefectural Univ Dept 1, Kyoto Prefectural Univ Dept 2, Kyoto Univ Dept 1, Kyushu Med Center, Kyushu Univ Dept 2, Matsushita Memorial Hosp, Matsuyama Red-Cross Hosp, Mitoyo General Hosp, Nagasaki Univ Dept 1, Nagasaki Univ Dept 2, Nagoya City Univ Dept 1, Nagoya Univ Dept 2, National Cancer Center, National Chiba Hosp, National Oita Hosp, National Osaka Hosp, National Tokyo Med Center, Nihon Kokan Hosp, Nihon Univ Dept 1, Nihon Univ Dept 3, Niigata Cancer Center, Niigata Univ Dept 1, Nippon Med School Dept 1, Ohmiya Med Center, Oita Med Coll Dept 1, Okayama Saiseikai Hosp, Okayama Univ Dept 1, Okayama Univ Dept 2, Osaka City Univ Dept 1, Osaka City Univ Dept 2, Osaka Med Coll Dept of Surg, Osaka Post Hosp, Osaka Univ Dept 2, Saitama Chuo Hosp, Saitama Med Coll Dept 1, Santoyo Sogo Hosp, Sapporo Keiyu Hosp, Sapporo Kosei Hosp, Shiga Med Coll Dept 1, Shikoku Cancer Center, Shinshu Univ Dept 1, Shinshu Univ Dept 2, St. Mary Med Coll Dept 1, St. Mary Med Coll Yokohama Hosp, Teikyo Univ Dept 1, Teikyo Univ Dept 2, Teikyo Univ Mizonokuchi Hosp, Tochigi Cancer Center, Tohoku Rosai Hosp, Tohoku Univ Dept 1, Tokushima Univ Dept 1, Tokyo Jikei Med Coll Dept 2, Tokyo Jikei Med Coll Aoto Hosp, Tokyo Metropolitan Komagome Hosp, Tokyo Med Coll Dept 3, Tokyo Med Dent Univ Dept 1, Tokyo Univ Med Institute, Tokyo Women's Med Coll Dept 2, Tokyo Women's Med Coll 2-Hosp, Tokyo Women's Med Coll GI Center, Toranomon Hosp, Tottori Univ Dept 1, Toyama Med Pharm Univ Dept 2, Tsuyama Chuo Hosp, Wakayama Med Coll Dept 2, Yamagata Chuo Hosp, Yamagata Kahoku Hosp, Yamanashi Univ Dept 1.

\section{Materials and methods}

In 1989, the registration committee of the Japanese Research Society for Gastric Cancer started to design and organize a personal computer registration system for patients with gastric cancer. The committee had to produce four programs for different computer operation systems: MS-DOS Basic, FileMaker Pro for Windows, FileMaker Pro for Macintosh, and a special database system sold in the Japanese market. Each member hospital selected an appropriate program for its computer. In 1991, the new nationwide registry was started. Definition and documentation were based on the Japanese Classification of Gastric Carcinoma, 11th edition [2]. The data list is shown in Table 1. Data of patients treated in 1991 were collected in 1998-1999 because 5-year follow-up data and the causes of death were requested to be reported. Names of patients and other personal data were removed from the data for privacy protection. The $3.5^{\prime \prime}$ floppy disks containing the data were mailed to the data center located in the National Cancer Center Hospital, Tokyo.

Eventually, data from 8851 patients were collected from 113 hospitals. Of these, 305 patients were excluded because of the final diagnosis as a recurrence in the gastric remnants, or histological diagnosis of other malignant tumors such as malignant lymphoma or leiomyosarcoma. Data of 238 patients were also excluded because of lack of essential data. Data from the remaining 8308 patients with primary gastric carcinoma underwent the final statistical analysis. The cumulative 5-year survival rate (5YSR) was calculated for various subsets of patients. Any types of death observed during 5 postoperative years, including direct death, or death due to other cancer or other diseases, were reflected in the survival analysis. Causes of death were also reported.

In this report, the data were translated into the 2 nd English edition of the Japanese Classification of Gastric Carcinoma [3], published in 1998, to facilitate international understanding. Although the UICC TNM Classification, 4th edition [4], published in 1987, was designed using the same principle as the Japanese classification,

Table 1. Collected data

Patient factors
1 year of treatment
2 registration no.
3 sex
4 date of birth
5 category of diseases
6 co-existing diseases
7 double (secondary) cancers
8 symptoms
9 preop. complications

Clinical findings

10 location

11 macroscopic type

12 depth of invasion (cT)

Surgical findings

13 date of surgery

14 macroscopic type

15 location

16 depth of invasion (sT)

17 organs of $\mathrm{T} 4$

18 lymph node metastasis (sN)

19 peritoneal metastasis ( $\mathrm{sP}$ )

20 liver metastasis $(\mathrm{sH})$

21 distant metastasis (sM)

22 stage (Japanese)

23 stage (UICC TNM)

Surgical treatment

24 approach

25 resection method

26 proximal margin (sPM)

27 distal margin (sDM)

28 lymph node dissection (D)

29 combined resection

30 curability

31 stage (Japanese)

\section{2 stage (UICC TNM)}

33 reconstruction

Histopathological findings
34 histological findings
35 cancer-stroma reaction
36 infiltration pattern (INF)
37 lymphatic invasion (ly)
38 venous invasion (v)
39 macroscopic type
40 depth of invasion (pT)
41 lymph node metastasis (pN)

Final findings

42 macroscopic type

43 depth of invasion (T)

44 lymph node metastasis (N)

45 hepatic metastasis $(\mathrm{H})$

46 peritoneal metastasis $(\mathrm{P})$

47 distant metastasis (M)

48 maximal diameter

49 proxim. resection margin (PM)

50 distal resection margin (DM)

51 curative potential of resection

52 stage (Japanese)

53 stage (UICC TNM)

Adjuvant treatments

54 preop. chemotherapy

55 intraop. chemotherapy

56 postop. chemotherapy

57 preop. treatments

58 intraop. treatments

59 postop. treatments

Treatment results

60 postop. complications

61 cause of death, or alive

62 last confirmed date alive 
the latter was more detailed in several categories. The Japanese T-category and staging system were compatible with the TNM classification. Peritoneal metastasis and liver metastasis were separately recorded in the Japanese classification as $\mathrm{P}$ - and $\mathrm{H}$-categories, but they could easily be translated into the M-category in the TNM classification. The $\mathrm{N}$-category was the most problematic for translation because the Japanese was based on the anatomical lymphatic streams and extension of node metastasis in association with location of the primary tumor, while the $\mathrm{N}$ category in the TNM classification was 4th edition also adopted the anatomical extension of node metastasis but not strictly based on lymph node (LN) stations as defined by the Japanese classification. Survival data stratified by TNM stage in this report should therefore be considered as preliminary.

The following data by important categories were shown on each table: total number of patients, direct death within 30 postoperative days, lost to follow-up, cumulative survival rates by year, $5 \%$ standard difference of 5YSR, number of alive patients at 5th postoperative year, causes of death such as local recurrence including lymph node metastasis, peritoneal recurrence, liver recurrence, distant metastasis excluding peritoneal and liver recurrence, recurrence of unknown site, other cancer, other disease, cause of death unknown. Cumulative survival curves of essential categories are also shown in Figs. 1-9. Definitions used in the Japanese Classification of Gastric Carcinoma, 11th edition, are briefly explained in the footnotes with reference to the 2nd English edition.

\section{Results}

Of the 8308 patients, 7935 underwent gastric resection, and the resection rate was $95.5 \%$. Eighty-seven patients died within 30 days of surgery, and the direct death rate was $1.0 \%(87 / 8308)$. The number of patients who were lost to follow-up was 571, and the percentage was $6.9 \%$ (Table 2).

For patients treated by gastric resection, the most frequent cause of death was peritoneal recurrence $(n=737)$ followed by other diseases $(n=390)$, local recurrence including node metastasis $(n=318)$, liver metastasis $(n=318)$, recurrence of unknown site $(n=266)$, distant metastasis other than the peritoneum and liver $(n=108)$, and secondary cancer $(n=104)$, while the cause of death was unknown in 131 patients (Table 2).

A high incidence of early-stage cancer is the major characteristic of this series, and the proportion of pT1 was $48.8 \%$ (3871/7935). The 5YSR of this population was $90.4 \%$ (Table 13). It was noteworthy that their primary cause of death was not cancer recurrence $(n=$
$84)$ but other diseases $(n=161)$. A large proportion of patients $(58.1 \%)$ had a stage-I disease by the Japanese classification, with the 5YSR of $89.9 \%$. Stage IV was found among $15.1 \%$ of the series, with a poor 5YSR of $9.0 \%$ (Table 38 ).

The proportion of upper-third cancer was $20.8 \%$ (1652/7935), and its 5YSR was relatively low at 57.1\%, The 5YSR of patients with esophageal invasion was even lower, at $33.4 \%$ (Table 7).

As for the histopathological type, poorly differentiated carcinoma (5YSR was $59.0 \%$ for solid type and $55.5 \%$ for nonsolid type) and mucinous carcinoma (5YSR: 49.7\%) showed poorer prognosis (Table 20). Also carcinoma with scirrhous type stroma (5YSR: $47.2 \%$ ), strong infiltrating growth or INF $\gamma$, (5YSR: $47.5 \%)$, marked lymphatic invasion or ly3, (5YSR: $24.7 \%$ ), and severe venous invasion or v3, (5YSR: $23.5 \%$ ) were other histopathological signs of poor prognosis (Tables 21-25).

D2 lymph node dissection was most commonly performed $(n=5366,67.6 \%)$ and its 5 YSR was $73.9 \%$. Almost $10 \%$ of the series $(783 / 7935)$ were treated by more extended node dissection, dissecting lymph nodes along the hepatoduodenal ligament (D3) and paraaortic region (D4). 5YSR of this subset was $51.2 \%$ and was poorer than that of D2. The result most likely reflects the generally advanced stage of the patients (Table 34). For pT2 cancer, 5YSR was higher among patients receiving systematic LN dissection (D2-D4) $(68.4 \%)$ than in those with limited dissection (D0 or D1) $(40.6 \%)$ (Tables 14, 15). Likewise, patients treated with systematic lymphadenectomy had higher 5YSR than those treated with limited dissection for pT3 cancer $(39.0 \%$ versus $8.0 \%$ ) (Tables 14,15$)$. Similarly in patients with pN1 metastasis, D2-D4 dissection showed better survival (5YSR: $62.3 \%$ ) to D0 or D1 (5YSR: $33.1 \%$ ) (Tables 27, 28).

The proportion of patients treated by combined resection of neighboring organs was 30.2\% (2394/7935), and the 5YSR was $49.3 \%$ (Table 35 ). The proportion of total gastrectomy was $30.7 \%$ (2439/7935), and its 5YSR was $49.2 \%$ (Table 32 ).

The curative potential of resection was an important prognostic factor. The number of patients with a high probability of cure (curability A) was 4959 (proportion: $62.5 \%$ ), and its 5 YSR was $88.5 \%$. On the other hand, patients with definite residual tumor (curability C) was 1235 (proportion: $15.6 \%$ ), and its 5YSR was $6.5 \%$ (Table 37).

\section{Discussion}

The data presented in this report were collected from 113 leading hospitals in Japan. The number of new pa- 
tients with gastric cancer in 1991 was estimated to be approximately 100000 ; thus the patients registered in this study $(n=8308)$ correspond to $8.3 \%$ of the total new patients. However, the data may not be suitable to use for epidemiological studies because they represent gastric cancer diagnosed and treated at specialized centers.

The findings were recorded based on the 11th edition of the Japanese Classification of Gastric Carcinoma (published in 1985). For international understanding, the registration committee tried to translate the data for the 2nd English edition of the Japanese classification (1998) and the 4th edition of the UICC TNM classification (1987). All data except the N-category were completely translatable. However, several considerations were necessary in translating the N-category. For example, regional LN stations, classified into four compartments (N1-N4) in the 11th Japanese edition, were reclassified into three compartments $(\mathrm{N} 1-\mathrm{N} 3)$ in the 2nd English edition. Consequently, metastases to the para-aortic nodes, classified as N4 in the 11th Japanese edition, are now included in N3 in the 2nd English edition. In this article, patients with $\mathrm{N} 3$ and N4 status according to the Japanese classification 11th edition and those treated by D3 or D4 dissection were analyzed together, and these groups were designated as N3 + N4 and D3+ D4.

The predominance of early-stage cancer with excellent survival, relative rarity of proximal cancer, and a high percentage of patients undergoing D2 and extended D3 + D4 dissection are among some features of this population that may seem strikingly different from the situation in the West. From the viewpoint of morbidity associated with surgery, it is important to note that combined resection of the pancreas was no longer a commonly performed procedure, 9.1\% (726/7935). Locoregional recurrence was not a common pattern of disease failure in this population, and this may reflect the effect of D2 dissection in terms of local control. However, differences in survival between the D2-D4 group and the limited surgery (D0, D1) group for pT2, pT3, and pN1 cancers may need some explanation. Death due to other diseases was observed in 10\% (25/ $239)$ of patients with pT2 cancer treated by limited surgery as compared with $4.3 \%(74 / 1714)$ in those treated with D2-D4 lymphadenectomy, and there is a possibility that limited lymphadenectomy had been selected for patients with co-morbidity who were destined to have a poor outcome. Peritoneal recurrence was observed among $37.7 \%(119 / 316)$ of pT3 cancer treated with limited surgery versus $22.6 \%$ (288/1274) treated with D2D4. There is a possibility that limited surgery might be selected for patients who had concomitant peritoneal seeding and nevertheless underwent gastrectomy. To clarify the survival benefit of systematic LN dissection
(D2-D4), we are now planning a multivariate analysis using the series.

Acknowledgments The Japanese Gastric Cancer Association Registration Committee appreciates very much the great effort of member hospitals in collecting accurate and detailed data for this project. We also wish to thank Dr. Peter Gunven, Karolinska University Hospital, Sweden, for his valuable assistance.

\section{References}

1. Japanese Research Society for Gastric Cancer and Japanese Gastric Cancer Association. Statistical report of nationwide registry of gastric carcinoma, No. 1-54 (in Japanese). Tokyo: National Cancer Center; 1972-2001.

2. Japanese Research Society for Gastric Cancer. General rules for gastric cancer study. 11th ed (in Japanese). Tokyo: Kanehara; 1985.

3. Japanese Gastric Cancer Association, Japanese classification of gastric carcinoma. 2nd English ed. Gastric Cancer 1998;1:10-24.

4. International Union Against Cancer. TNM classification of malignant tumours. 4th ed. Hermanek P, Sobin LH, editors. Berlin, Heidelberg, New York, London, Paris, Tokyo: Springer; 1987.

5. Japanese Gastric Cancer Association, Japanese classification of gastric carcinoma. 12th ed (in Japanese). Tokyo: Kanehara; 1993. 
Table 2. Primary cancer and resected cases

\begin{tabular}{|c|c|c|c|c|c|c|c|c|c|c|c|c|c|c|c|c|c|c|}
\hline categories & $\begin{array}{c}\text { total } \\
\text { number }\end{array}$ & $\begin{array}{l}\text { direct } \\
\text { death }\end{array}$ & $\begin{array}{l}\text { lost } \\
\text { f.u. }\end{array}$ & $\begin{array}{l}1 \mathrm{ysr} \\
(\%)\end{array}$ & $\begin{array}{l}2 y s r \\
(\%)\end{array}$ & $\begin{array}{c}3 \text { ysr } \\
(\%)\end{array}$ & $\begin{array}{l}4 \mathrm{ysr} \\
(\%)\end{array}$ & $\begin{array}{l}5 \text { ysr } \\
(\%)\end{array}$ & $\begin{array}{l}5 \% \\
\text { s.d. }\end{array}$ & alive & $\begin{array}{c}\text { local } \\
\text { rec. }\end{array}$ & $\begin{array}{l}\text { peri- } \\
\text { toneal }\end{array}$ & $\begin{array}{c}\text { liver } \\
\text { rec. }\end{array}$ & $\begin{array}{c}\text { distant } \\
\text { meta. }\end{array}$ & $\mathrm{R}$ & $\begin{array}{l}\text { other } \\
\text { cancer }\end{array}$ & $\begin{array}{c}\text { other } \\
\text { disease }\end{array}$ & $\begin{array}{c}\text { un- } \\
\text { known }\end{array}$ \\
\hline Primary cancer & 8308 & 87 & 571 & 83.9 & 75.4 & 70.8 & 68.0 & 66.0 & 1.1 & 5019 & 336 & 876 & 315 & 126 & 316 & 109 & 415 & 138 \\
\hline Unresected cases & 7935 & 81 & 548 & 86.4 & 78.0 & 73.2 & 70.4 & 68.2 & 1.1 & 4966 & 318 & 737 & 286 & 108 & 266 & 104 & 390 & 131 \\
\hline Resected cases & 240 & 6 & 12 & 16.0 & 4.5 & 3.5 & 2.4 & 2.4 & 2.2 & 4 & 16 & 114 & 17 & 13 & 37 & 2 & 13 & 6 \\
\hline Other surgeries & 133 & 0 & 11 & 56.8 & 48.6 & 45.3 & 43.6 & 42.7 & 8.8 & 49 & 2 & 25 & 12 & 5 & 13 & 3 & 12 & 1 \\
\hline
\end{tabular}

\section{Abbreviations for tables}

lost f.u.

ysr (\%)

$5 \%$ s.d.

local rec.

peritoneal

liver rec.

distant meta.

$\mathbf{R}$

unknown

$\begin{array}{ll} & \text { Abbreviations for figures } \\ \text { dd } & \text { patients by direct death } \\ \text { lof } & \text { lost to follow-up }\end{array}$

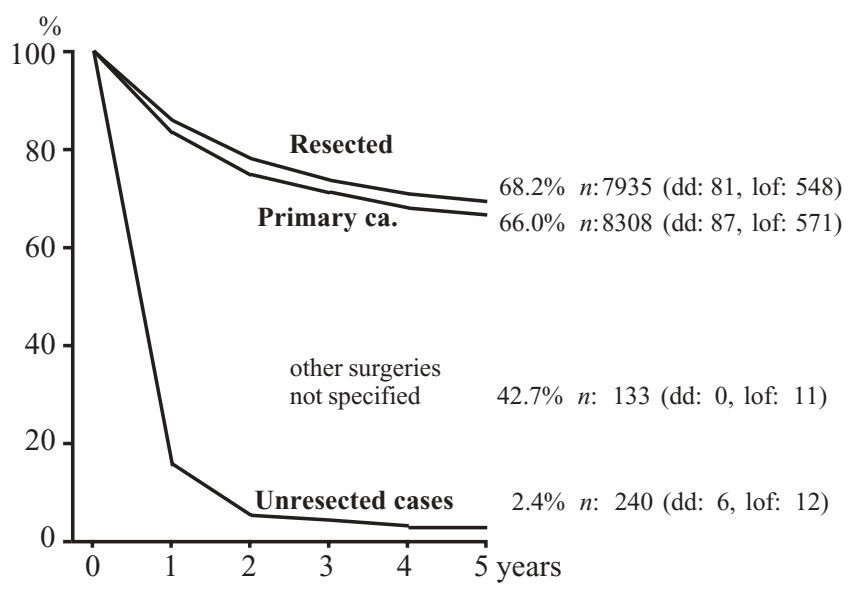

Fig. 1. Primary cancer and resected cases

Table 3. Sex (resected cases)

\begin{tabular}{|c|c|c|c|c|c|c|c|c|c|c|c|c|c|c|c|c|c|c|}
\hline categories & $\begin{array}{c}\text { total } \\
\text { number }\end{array}$ & $\begin{array}{l}\text { direct } \\
\text { death }\end{array}$ & $\begin{array}{l}\text { lost } \\
\text { f.u. }\end{array}$ & $\begin{array}{l}1 \mathrm{ysr} \\
(\%)\end{array}$ & $\begin{array}{l}2 \mathrm{ysr} \\
(\%)\end{array}$ & $\begin{array}{r}3 \text { ysr } \\
(\%)\end{array}$ & $\begin{array}{l}4 \mathrm{ysr} \\
(\%)\end{array}$ & $\begin{array}{l}5 \mathrm{ysr} \\
(\%)\end{array}$ & $\begin{array}{l}5 \% \\
\text { s.d. }\end{array}$ & alive & $\begin{array}{l}\text { local } \\
\text { rec. }\end{array}$ & $\begin{array}{l}\text { peri- } \\
\text { toneal }\end{array}$ & $\begin{array}{l}\text { liver } \\
\text { rec. }\end{array}$ & $\begin{array}{l}\text { distant } \\
\text { meta. }\end{array}$ & $\mathrm{R}$ & $\begin{array}{l}\text { other } \\
\text { cancer }\end{array}$ & $\begin{array}{c}\text { other } \\
\text { disease }\end{array}$ & $\begin{array}{c}\text { un- } \\
\text { known }\end{array}$ \\
\hline Male & 5493 & 70 & 394 & 85.6 & 77.1 & 72.1 & 69.4 & 67.2 & 1.3 & 3368 & 227 & 465 & 223 & 76 & 187 & 87 & 295 & 101 \\
\hline Female & 2441 & 11 & 154 & 88.2 & 79.9 & 75.5 & 72.6 & 70.6 & 1.9 & 1597 & 91 & 272 & 63 & 32 & 79 & 17 & 95 & 30 \\
\hline Not specified & 1 & 0 & 0 & 100.0 & 100.0 & 100.0 & 100.0 & 100.0 & 0.0 & 1 & 0 & 0 & 0 & 0 & 0 & 0 & 0 & 0 \\
\hline
\end{tabular}

Table 4. Age (resected cases)

\begin{tabular}{|c|c|c|c|c|c|c|c|c|c|c|c|c|c|c|c|c|c|c|}
\hline categories & $\begin{array}{c}\text { total } \\
\text { number }\end{array}$ & $\begin{array}{l}\text { direct } \\
\text { death }\end{array}$ & $\begin{array}{l}\text { lost } \\
\text { f.u. }\end{array}$ & $\begin{array}{l}1 \text { ysr } \\
(\%)\end{array}$ & $\begin{array}{l}2 \mathrm{ysr} \\
(\%)\end{array}$ & $\begin{array}{r}3 \text { ysr } \\
(\%)\end{array}$ & $\begin{array}{l}4 \mathrm{ysr} \\
(\%)\end{array}$ & $\begin{array}{l}5 \text { ysr } \\
(\%)\end{array}$ & $\begin{array}{l}5 \% \\
\text { s.d. }\end{array}$ & alive & $\begin{array}{l}\text { local } \\
\text { rec. }\end{array}$ & $\begin{array}{l}\text { peri- } \\
\text { toneal }\end{array}$ & $\begin{array}{l}\text { liver } \\
\text { rec. }\end{array}$ & $\begin{array}{l}\text { distant } \\
\text { meta. }\end{array}$ & $\mathrm{R}$ & $\begin{array}{l}\text { other } \\
\text { cancer }\end{array}$ & $\begin{array}{c}\text { other } \\
\text { disease }\end{array}$ & $\begin{array}{c}\text { un- } \\
\text { known }\end{array}$ \\
\hline-39 & 302 & 0 & 23 & 87.5 & 79.2 & 76.7 & 74.6 & 72.4 & 5.3 & 199 & 6 & 53 & 2 & 4 & 11 & 0 & 2 & 2 \\
\hline $40-59$ & 2805 & 14 & 182 & 89.9 & 83.6 & 79.9 & 77.4 & 76.1 & 1.6 & 1976 & 113 & 240 & 83 & 42 & 57 & 16 & 60 & 22 \\
\hline 60-79 & 4276 & 61 & 292 & 84.6 & 75.3 & 70.2 & 67.1 & 64.8 & 1.5 & 2537 & 170 & 404 & 179 & 55 & 157 & 78 & 258 & 85 \\
\hline $80-$ & 344 & 6 & 32 & 74.5 & 60.3 & 49.3 & 45.1 & 39.7 & 5.5 & 119 & 23 & 31 & 15 & 6 & 24 & 8 & 63 & 17 \\
\hline Not specified & 207 & 0 & 19 & 93.0 & 82.2 & 76.4 & 75.9 & 72.1 & 6.5 & 134 & 6 & 9 & 7 & 1 & 17 & 2 & 7 & 5 \\
\hline
\end{tabular}

Table 5. Age in males (resected cases)

\begin{tabular}{|c|c|c|c|c|c|c|c|c|c|c|c|c|c|c|c|c|c|c|}
\hline categories & $\begin{array}{c}\text { total } \\
\text { number }\end{array}$ & $\begin{array}{l}\text { direct } \\
\text { death }\end{array}$ & $\begin{array}{l}\text { lost } \\
\text { f.u. }\end{array}$ & $\begin{array}{l}1 \mathrm{ysr} \\
(\%)\end{array}$ & $\begin{array}{l}2 \text { ysr } \\
(\%)\end{array}$ & $\begin{array}{r}3 \text { ysr } \\
(\%)\end{array}$ & $\begin{array}{l}4 \text { ysr } \\
(\%)\end{array}$ & $\begin{array}{l}5 \mathrm{ysr} \\
(\%)\end{array}$ & $\begin{array}{l}5 \% \\
\text { s.d. }\end{array}$ & alive & $\begin{array}{l}\text { local } \\
\text { rec. }\end{array}$ & $\begin{array}{l}\text { peri- } \\
\text { toneal }\end{array}$ & $\begin{array}{l}\text { liver } \\
\text { rec. }\end{array}$ & $\begin{array}{c}\text { distant } \\
\text { meta. }\end{array}$ & $\mathrm{R}$ & $\begin{array}{l}\text { other } \\
\text { cancer }\end{array}$ & $\begin{array}{c}\text { other } \\
\text { disease }\end{array}$ & $\begin{array}{c}\text { un- } \\
\text { known }\end{array}$ \\
\hline-39 & 150 & 0 & 14 & 87.8 & 81.5 & 78.7 & 76.5 & 75.0 & 7.2 & 100 & 3 & 19 & 1 & 3 & 8 & 0 & 0 & 2 \\
\hline $40-59$ & 1964 & 11 & 138 & 90.1 & 84.0 & 80.1 & 77.7 & 76.4 & 2.0 & 1381 & 84 & 143 & 67 & 28 & 37 & 13 & 47 & 15 \\
\hline $60-79$ & 3006 & 53 & 206 & 83.1 & 73.4 & 68.2 & 65.3 & 62.7 & 1.8 & 1721 & 118 & 278 & 140 & 40 & 115 & 69 & 201 & 65 \\
\hline $80-$ & 224 & 6 & 22 & 73.3 & 60.7 & 49.3 & 44.7 & 40.0 & 6.9 & 77 & 16 & 18 & 9 & 4 & 15 & 3 & 40 & 14 \\
\hline Not specified & 148 & 0 & 14 & 90.9 & 79.3 & 71.8 & 71.1 & 66.6 & 8.1 & 88 & 6 & 7 & 6 & 1 & 12 & 2 & 7 & 5 \\
\hline
\end{tabular}


Table 6. Age in females (resected cases)

\begin{tabular}{|c|c|c|c|c|c|c|c|c|c|c|c|c|c|c|c|c|c|c|}
\hline categories & $\begin{array}{c}\text { total } \\
\text { number }\end{array}$ & $\begin{array}{l}\text { direct } \\
\text { death }\end{array}$ & $\begin{array}{l}\text { lost } \\
\text { f.u. }\end{array}$ & $\begin{array}{l}1 \mathrm{ysr} \\
(\%)\end{array}$ & $\begin{array}{l}2 \mathrm{ysr} \\
(\%)\end{array}$ & $\begin{array}{l}3 \text { ysr } \\
(\%)\end{array}$ & $\begin{array}{l}4 \mathrm{ysr} \\
(\%)\end{array}$ & $\begin{array}{l}5 y s r \\
(\%)\end{array}$ & $\begin{array}{l}5 \% \\
\text { s.d. }\end{array}$ & alive & $\begin{array}{l}\text { local } \\
\text { rec. }\end{array}$ & $\begin{array}{l}\text { peri- } \\
\text { toneal }\end{array}$ & $\begin{array}{l}\text { liver } \\
\text { rec. }\end{array}$ & $\begin{array}{c}\text { distant } \\
\text { meta. }\end{array}$ & $\mathrm{R}$ & $\begin{array}{l}\text { other } \\
\text { cancer }\end{array}$ & $\begin{array}{l}\text { other } \\
\text { disease }\end{array}$ & $\begin{array}{c}\text { un- } \\
\text { known }\end{array}$ \\
\hline-39 & 152 & 0 & 9 & 87.3 & 76.9 & 74.8 & 72.7 & 69.8 & 7.6 & 99 & 3 & 34 & 1 & 1 & 3 & 0 & 2 & 0 \\
\hline $40-59$ & 841 & 3 & 44 & 89.4 & 82.6 & 79.6 & 76.8 & 75.3 & 3.0 & 595 & 29 & 97 & 16 & 14 & 20 & 3 & $1 \overline{3}$ & 7 \\
\hline $60-79$ & 1270 & 8 & 86 & 88.2 & 80.0 & 74.8 & 71.6 & 69.8 & 2.6 & 816 & 52 & 126 & 39 & 15 & 42 & 9 & 57 & 20 \\
\hline $80-$ & 120 & 0 & 10 & 76.7 & 59.5 & 49.4 & 45.7 & 39.1 & 9.3 & 42 & 7 & 13 & 6 & 2 & 9 & 5 & 23 & 3 \\
\hline Not specified & 58 & 0 & 5 & 98.2 & 89.1 & 87.3 & 87.3 & 85.4 & 9.5 & 45 & 0 & 2 & 1 & 0 & 5 & 0 & 0 & 0 \\
\hline
\end{tabular}

Table 7. Tumor location (resected cases)

\begin{tabular}{|c|c|c|c|c|c|c|c|c|c|c|c|c|c|c|c|c|c|c|}
\hline categories & $\begin{array}{c}\text { total } \\
\text { number }\end{array}$ & $\begin{array}{l}\text { direct } \\
\text { death }\end{array}$ & $\begin{array}{l}\text { lost } \\
\text { f.u. }\end{array}$ & $\begin{array}{l}1 \mathrm{ysr} \\
(\%)\end{array}$ & $\begin{array}{l}2 \text { ysr } \\
(\%)\end{array}$ & $\begin{array}{r}3 \text { ysr } \\
(\%)\end{array}$ & $\begin{array}{l}4 \text { ysr } \\
(\%)\end{array}$ & $\begin{array}{l}5 \mathrm{ysr} \\
(\%)\end{array}$ & $\begin{array}{l}5 \% \\
\text { s.d. }\end{array}$ & alive & $\begin{array}{l}\text { local } \\
\text { rec. }\end{array}$ & $\begin{array}{l}\text { peri- } \\
\text { toneal }\end{array}$ & $\begin{array}{l}\text { liver } \\
\text { rec. }\end{array}$ & $\begin{array}{l}\text { distant } \\
\text { meta. }\end{array}$ & $\mathrm{R}$ & $\begin{array}{l}\text { other } \\
\text { cancer }\end{array}$ & $\begin{array}{c}\text { other } \\
\text { disease }\end{array}$ & $\begin{array}{c}\text { un- } \\
\text { known }\end{array}$ \\
\hline & 1643 & 29 & 124 & 81.2 & 68.1 & 62.4 & 59.5 & 56.9 & 2.5 & 845 & 90 & 212 & 69 & 35 & 80 & 18 & 112 & 29 \\
\hline & & 28 & 193 & 88.1 & 82.2 & 78.4 & 76.2 & 74.4 & 1.6 & 2246 & 83 & 27 & 81 & 4 & 90 & 34 & & 43 \\
\hline Lowe & 3047 & 24 & 231 & 87.3 & 78.6 & 73.2 & 69.8 & 67.6 & 1.7 & 1874 & 145 & 252 & 136 & 32 & 96 & 52 & 146 & 59 \\
\hline Esophageal invasion & 313 & 5 & 21 & 68.1 & 45.5 & 37.5 & 33.9 & 32.8 & 5.5 & 91 & 27 & 57 & 19 & 18 & 36 & 0 & 31 & 8 \\
\hline Not specified & 1 & 0 & 0 & 100.0 & 100.0 & 100.0 & 100.0 & 100.0 & 0.0 & 1 & 0 & 0 & 0 & 0 & 0 & 0 & 0 & 0 \\
\hline
\end{tabular}

Table 8. Tumor location, circumference (resected cases)

\begin{tabular}{|c|c|c|c|c|c|c|c|c|c|c|c|c|c|c|c|c|c|c|}
\hline categories & $\begin{array}{c}\text { total } \\
\text { number }\end{array}$ & $\begin{array}{l}\text { direct } \\
\text { death }\end{array}$ & $\begin{array}{l}\text { lost } \\
\text { f.u. }\end{array}$ & $\begin{array}{l}1 \text { ysr } \\
(\%)\end{array}$ & $\begin{array}{l}2 \mathrm{ysr} \\
(\%)\end{array}$ & $\begin{array}{l}3 \text { ysr } \\
(\%)\end{array}$ & $\begin{array}{l}4 \mathrm{ysr} \\
(\%)\end{array}$ & $\begin{array}{l}5 \text { ysr } \\
(\%)\end{array}$ & $\begin{array}{l}5 \% \\
\text { s.d. }\end{array}$ & alive & $\begin{array}{l}\text { local } \\
\text { rec. }\end{array}$ & $\begin{array}{l}\text { peri- } \\
\text { toneal }\end{array}$ & $\begin{array}{l}\text { liver } \\
\text { rec. }\end{array}$ & $\begin{array}{l}\text { distant } \\
\text { meta. }\end{array}$ & $\mathrm{R}$ & $\begin{array}{l}\text { other } \\
\text { cancer }\end{array}$ & $\begin{array}{l}\text { other } \\
\text { disease }\end{array}$ & $\begin{array}{c}\text { un- } \\
\text { known }\end{array}$ \\
\hline Lesser curvature & 3141 & 36 & 224 & 88.9 & 81.6 & 77.0 & 74.2 & 71.6 & 1.6 & 2063 & 112 & 204 & 103 & 42 & 98 & 44 & 163 & 52 \\
\hline Greater curvature & 1606 & 14 & 104 & 90.8 & 84.6 & 80.2 & 78.1 & 76.1 & 2.2 & 1132 & 41 & 102 & 48 & 17 & 35 & 20 & 74 & 19 \\
\hline Anterior wall & 1259 & 7 & 76 & 91.8 & 85.6 & 81.2 & 78.6 & 76.8 & 2.4 & 901 & 29 & 64 & 46 & 12 & 27 & 22 & 57 & 18 \\
\hline Posterior wall & 1000 & 7 & 78 & 89.0 & 81.2 & 77.8 & 75.1 & 73.7 & 2.9 & 670 & 21 & 75 & 38 & 10 & 29 & 12 & 40 & 20 \\
\hline Circumferential & 927 & 17 & 66 & 60.0 & 39.2 & 30.9 & 26.7 & 24.4 & 2.9 & 198 & 115 & 292 & 51 & 27 & 77 & 6 & 56 & 22 \\
\hline Not specified & 2 & 0 & 0 & 100.0 & 100.0 & 100.0 & 100.0 & 100.0 & 0.0 & 2 & 0 & 0 & 0 & 0 & 0 & 0 & 0 & 0 \\
\hline
\end{tabular}

Table 9. Peritoneal metastasis (resected cases)

\begin{tabular}{|c|c|c|c|c|c|c|c|c|c|c|c|c|c|c|c|c|c|c|}
\hline categories & $\begin{array}{c}\text { total } \\
\text { number }\end{array}$ & $\begin{array}{l}\text { direct } \\
\text { death }\end{array}$ & $\begin{array}{l}\text { lost } \\
\text { f.u. }\end{array}$ & $\begin{array}{l}1 \mathrm{ysr} \\
(\%)\end{array}$ & $\begin{array}{l}2 \text { ysr } \\
(\%)\end{array}$ & $\begin{array}{r}3 \text { ysr } \\
(\%)\end{array}$ & $\begin{array}{l}4 \text { ysr } \\
(\%)\end{array}$ & $\begin{array}{l}5 y s r \\
(\%)\end{array}$ & $\begin{array}{l}5 \% \\
\text { s.d. }\end{array}$ & alive & $\begin{array}{c}\text { local } \\
\text { rec. }\end{array}$ & $\begin{array}{l}\text { peri- } \\
\text { toneal }\end{array}$ & $\begin{array}{l}\text { liver } \\
\text { rec. }\end{array}$ & $\begin{array}{c}\text { distant } \\
\text { meta. }\end{array}$ & $\mathrm{R}$ & $\begin{array}{l}\text { other } \\
\text { cancer }\end{array}$ & $\begin{array}{c}\text { other } \\
\text { disease }\end{array}$ & $\begin{array}{c}\text { un- } \\
\text { known }\end{array}$ \\
\hline P0 not metastatic & 7404 & 70 & 497 & 89.7 & 82.0 & 77.4 & 74.6 & 72.3 & 1.1 & 4938 & 297 & 441 & 258 & 94 & 214 & 104 & 366 & 125 \\
\hline P1 metastatic & 529 & 11 & 50 & 39.2 & 18.2 & 10.6 & 8.1 & 7.1 & 2.5 & 27 & 21 & 296 & 28 & 14 & 52 & 0 & 24 & 6 \\
\hline Not specified & 2 & 0 & 1 & 100.0 & 100.0 & 100.0 & 100.0 & 100.0 & 0.0 & 1 & 0 & 0 & 0 & 0 & 0 & 0 & 0 & 0 \\
\hline
\end{tabular}

Footnote for Table 7: Tumor location

The stomach is anatomically divided into three portions. If more than one portion is involved, all involved portions should be described in order of degree of involvement. Namely, the first letter indicates the portion in which the bulk of the tumor is situated, and the portion is used for categorization in Table 7.
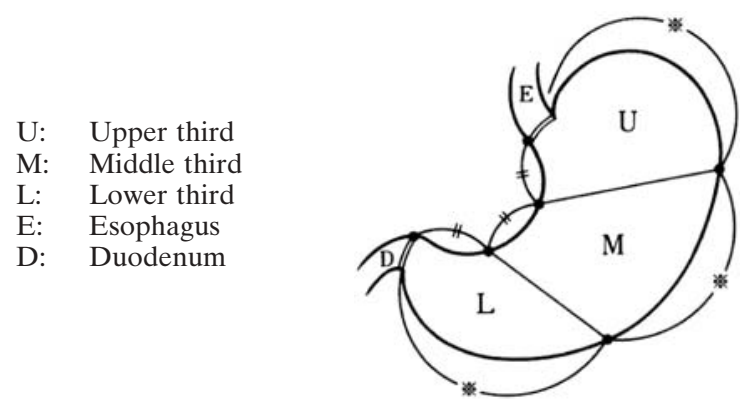

Footnote for Table 8: Tumor location, circumference

The cross-sectional circumference of the stomach is divided into four equal parts as shown in the figure below. Circumferential involvement is recorded as Circ.

Less: Lesser curvature

Gre: Greater curvature

Ant: Anterior wall

Post: Posterior wall

Circ: Circumferential

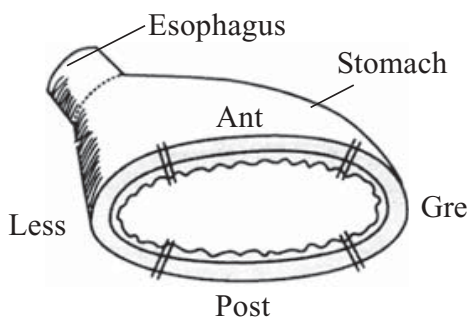

Footnote for Tables 9-11: Distant metastasis

Distant metastasis is separately documented by peritoneal metastasis ( $\mathrm{P} 0$ or $\mathrm{P} 1)$, liver metastasis ( $\mathrm{H} 0$ or $\mathrm{H} 1)$, and other distant metastases (M0 or M1). 
Table 10. Liver metastasis (resected cases)

\begin{tabular}{|c|c|c|c|c|c|c|c|c|c|c|c|c|c|c|c|c|c|c|}
\hline categories & $\begin{array}{c}\text { total } \\
\text { number }\end{array}$ & $\begin{array}{l}\text { direct } \\
\text { death }\end{array}$ & $\begin{array}{l}\text { lost } \\
\text { f.u. }\end{array}$ & $\begin{array}{l}1 \text { ysr } \\
(\%)\end{array}$ & $\begin{array}{l}2 \text { ysr } \\
(\%)\end{array}$ & $\begin{array}{l}3 \text { ysr } \\
(\%)\end{array}$ & $\begin{array}{l}4 \mathrm{ysr} \\
(\%)\end{array}$ & $\begin{array}{l}5 \text { ysr } \\
(\%)\end{array}$ & $\begin{array}{l}5 \% \\
\text { s.d. }\end{array}$ & alive & $\begin{array}{c}\text { local } \\
\text { rec. }\end{array}$ & $\begin{array}{l}\text { peri- } \\
\text { toneal }\end{array}$ & $\begin{array}{l}\text { liver } \\
\text { rec. }\end{array}$ & $\begin{array}{l}\text { distant } \\
\text { meta. }\end{array}$ & $\mathrm{R}$ & $\begin{array}{l}\text { other } \\
\text { cancer }\end{array}$ & $\begin{array}{c}\text { other } \\
\text { disease }\end{array}$ & $\begin{array}{c}\text { un- } \\
\text { known }\end{array}$ \\
\hline H0 not metastatic & 7404 & 70 & 497 & 89.7 & 82.0 & 77.4 & 74.6 & 72.3 & 1.1 & 4938 & 297 & 441 & 258 & 94 & 214 & 104 & 366 & 125 \\
\hline H1 metastatic & 271 & 9 & 16 & 31.1 & 11.9 & 7.2 & 5.5 & 4.6 & 2.7 & 10 & 14 & 41 & 136 & 2 & 22 & 0 & 16 & 5 \\
\hline Not specified & 2 & 0 & 1 & 100.0 & 100.0 & 100.0 & 100.0 & 100.0 & 0.0 & 1 & 0 & 0 & 0 & 0 & 0 & 0 & 0 & 0 \\
\hline
\end{tabular}

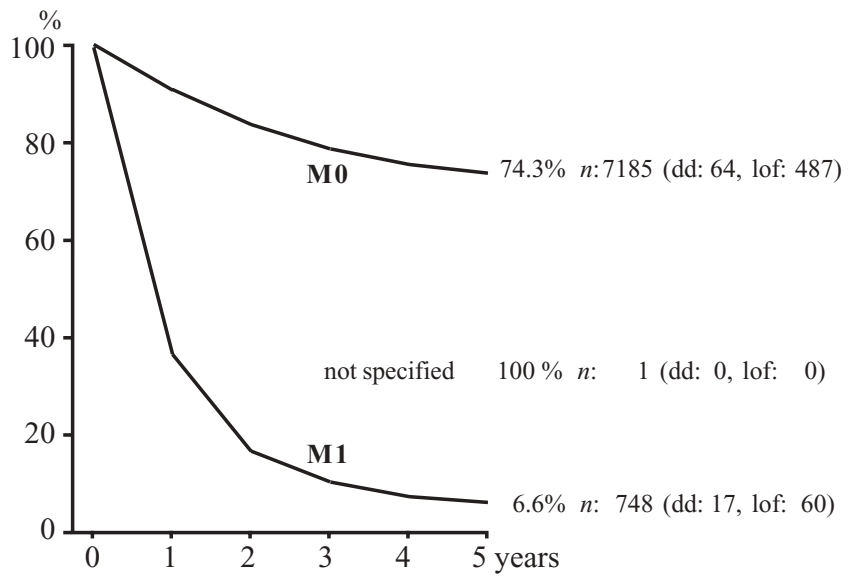

Fig. 2. Distant metastasis $(\mathrm{M})$ including $\mathrm{P}$ and $\mathrm{H}$

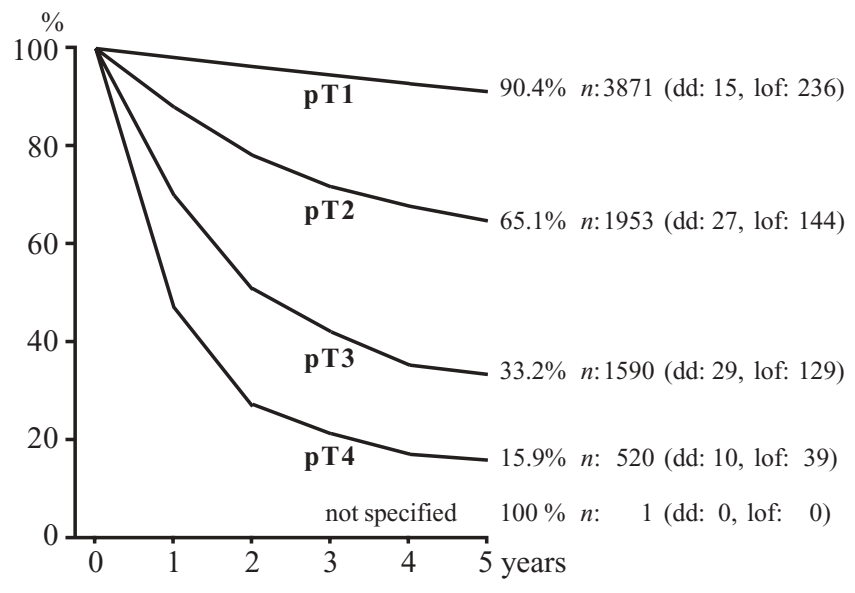

Fig. 3. Depth of invasion (pT)

Table 11. Distant metastasis including peritoneal and liver metastasis (resected cases)

\begin{tabular}{|c|c|c|c|c|c|c|c|c|c|c|c|c|c|c|c|c|c|c|}
\hline categories & $\begin{array}{c}\text { total } \\
\text { number }\end{array}$ & $\begin{array}{l}\text { direct } \\
\text { death }\end{array}$ & $\begin{array}{l}\text { lost } \\
\text { f.u. }\end{array}$ & $\begin{array}{l}1 \mathrm{ysr} \\
(\%)\end{array}$ & $\begin{array}{l}2 \text { ysr } \\
(\%)\end{array}$ & $\begin{array}{c}3 \text { ysr } \\
(\%)\end{array}$ & $\begin{array}{l}4 y s r \\
(\%)\end{array}$ & $\begin{array}{l}5 \text { ysr } \\
(\%)\end{array}$ & $\begin{array}{l}5 \% \\
\text { s.d. }\end{array}$ & alive & $\begin{array}{c}\text { local } \\
\text { rec. }\end{array}$ & $\begin{array}{l}\text { peri- } \\
\text { toneal }\end{array}$ & $\begin{array}{l}\text { liver } \\
\text { rec. }\end{array}$ & $\begin{array}{l}\text { distant } \\
\text { meta. }\end{array}$ & $\mathrm{R}$ & $\begin{array}{l}\text { other } \\
\text { cancer }\end{array}$ & $\begin{array}{c}\text { other } \\
\text { disease }\end{array}$ & $\begin{array}{c}\text { un- } \\
\text { known }\end{array}$ \\
\hline M0 not metastatic & 7185 & 64 & 487 & 91.3 & 84.0 & 79.4 & 76.6 & 74.3 & 1.1 & 4927 & 280 & 423 & 144 & 86 & 197 & 104 & 353 & 120 \\
\hline M1 metastatic & 748 & 17 & 60 & 38.4 & 17.3 & 10.1 & 8.0 & 6.6 & 2.0 & 38 & 38 & 314 & 142 & 22 & 69 & 0 & 37 & 11 \\
\hline Not specified & 2 & 0 & 1 & 100.0 & 100.0 & 100.0 & 100.0 & 100.0 & 0.0 & 1 & 0 & 0 & 0 & 0 & 0 & 0 & 0 & 0 \\
\hline
\end{tabular}

Table 12. Depth of invasion (resected cases)

\begin{tabular}{|c|c|c|c|c|c|c|c|c|c|c|c|c|c|c|c|c|c|c|}
\hline categories & $\begin{array}{c}\text { total } \\
\text { number }\end{array}$ & $\begin{array}{l}\text { direct } \\
\text { death }\end{array}$ & $\begin{array}{l}\text { lost } \\
\text { f.u. }\end{array}$ & $\begin{array}{l}1 \mathrm{ysr} \\
(\%)\end{array}$ & $\begin{array}{l}2 \mathrm{ysr} \\
(\%)\end{array}$ & $\begin{array}{c}3 \text { ysr } \\
(\%)\end{array}$ & $\begin{array}{l}4 \mathrm{ysr} \\
(\%)\end{array}$ & $\begin{array}{l}5 \text { ysr } \\
(\%)\end{array}$ & $\begin{array}{l}5 \% \\
\text { s.d. }\end{array}$ & alive & $\begin{array}{l}\text { local } \\
\text { rec. }\end{array}$ & $\begin{array}{l}\text { peri- } \\
\text { toneal }\end{array}$ & $\begin{array}{l}\text { liver } \\
\text { rec. }\end{array}$ & $\begin{array}{c}\text { distant } \\
\text { meta. }\end{array}$ & $\mathrm{R}$ & $\begin{array}{c}\text { other } \\
\text { cancer }\end{array}$ & $\begin{array}{c}\text { other } \\
\text { disease }\end{array}$ & $\begin{array}{c}\text { un- } \\
\text { known }\end{array}$ \\
\hline $\operatorname{Mucosa}(\mathrm{M})$ & 2209 & 4 & 118 & 98.0 & 96.6 & 95.2 & 94.0 & 92.5 & 1.1 & 1932 & 6 & 4 & 3 & 2 & 6 & 33 & 81 & 20 \\
\hline Submucosa (SM) & 1662 & 11 & 118 & 96.0 & 93.7 & 90.8 & 89.3 & 87.6 & 1.7 & 1346 & 17 & 14 & 17 & 7 & 8 & 28 & 80 & 16 \\
\hline Muscularis (MP) & 817 & 6 & 58 & 93.9 & 87.8 & 83.7 & 81.2 & 78.4 & 2.9 & 590 & 19 & 22 & 33 & 6 & 18 & 6 & 36 & 23 \\
\hline Subserosa (SS) & 1136 & 21 & 86 & 84.2 & 71.0 & 62.5 & 58.4 & 55.4 & 3.0 & 570 & 69 & 122 & 73 & 28 & 51 & 19 & 63 & 34 \\
\hline Serosa (SE) & 1590 & 29 & 129 & 70.0 & 50.6 & 41.7 & 36.1 & 33.2 & 2.5 & 457 & 147 & 407 & 117 & 51 & 113 & 17 & 93 & 30 \\
\hline $\begin{array}{l}\text { Adjacent structures } \\
\text { (SI) }\end{array}$ & 520 & 10 & 39 & 48.3 & 27.6 & 21.0 & 17.7 & 15.9 & 3.4 & 70 & 60 & 168 & 43 & 14 & 70 & 1 & 37 & 8 \\
\hline Not specified & 1 & 0 & 0 & 100.0 & 100.0 & 100.0 & 100.0 & 100.0 & 0.0 & 1 & 0 & 0 & 0 & 0 & 0 & 0 & 0 & 0 \\
\hline
\end{tabular}

Table 13. $\mathrm{pT}$ classification (resected cases)

\begin{tabular}{|c|c|c|c|c|c|c|c|c|c|c|c|c|c|c|c|c|c|c|}
\hline categories & $\begin{array}{c}\text { total } \\
\text { number }\end{array}$ & $\begin{array}{l}\text { direct } \\
\text { death }\end{array}$ & $\begin{array}{l}\text { lost } \\
\text { f.u. }\end{array}$ & $\begin{array}{l}1 \mathrm{ysr} \\
(\%)\end{array}$ & $\begin{array}{l}2 \mathrm{ysr} \\
(\%)\end{array}$ & $\begin{array}{l}3 \mathrm{ysr} \\
(\%)\end{array}$ & $\begin{array}{l}4 y s r \\
(\%)\end{array}$ & $\begin{array}{l}5 \text { ysr } \\
(\%)\end{array}$ & $\begin{array}{l}5 \% \\
\text { s.d. }\end{array}$ & alive & $\begin{array}{c}\text { local } \\
\text { rec. }\end{array}$ & $\begin{array}{l}\text { peri- } \\
\text { toneal }\end{array}$ & $\begin{array}{l}\text { liver } \\
\text { rec. }\end{array}$ & $\begin{array}{c}\text { distant } \\
\text { meta. }\end{array}$ & $\mathrm{R}$ & $\begin{array}{l}\text { other } \\
\text { cancer }\end{array}$ & $\begin{array}{c}\text { other } \\
\text { disease }\end{array}$ & $\begin{array}{c}\text { un- } \\
\text { known }\end{array}$ \\
\hline pT1 & 3871 & 15 & 236 & 97.1 & 95.4 & 93.3 & 92.0 & 90.4 & 1.0 & 3278 & 23 & 18 & 20 & 9 & 14 & 61 & 161 & 36 \\
\hline pT2 & 1953 & 27 & 144 & 88.3 & 78.1 & 71.5 & 68.0 & 65.1 & 2.2 & 1160 & 88 & 144 & 106 & 34 & 69 & 25 & 99 & 57 \\
\hline pT3 & 1590 & 29 & 129 & 70.0 & 50.6 & 41.7 & 36.1 & 33.2 & 2.5 & 457 & 147 & 407 & 117 & 51 & 113 & 17 & 93 & 30 \\
\hline pT4 & 520 & 10 & 39 & 48.3 & 27.6 & 21.0 & 17.7 & 15.9 & 3.4 & 70 & 60 & 168 & 43 & 14 & 70 & 1 & 37 & 8 \\
\hline Not specified & 1 & 0 & 0 & 100.0 & 100.0 & 100.0 & 100.0 & 100.0 & 0.0 & 1 & 0 & 0 & 0 & 0 & 0 & 0 & 0 & 0 \\
\hline
\end{tabular}


Table 14. pT classification by D0 or D1 dissection (resected cases)

\begin{tabular}{|c|c|c|c|c|c|c|c|c|c|c|c|c|c|c|c|c|c|c|}
\hline categories & $\begin{array}{c}\text { total } \\
\text { number }\end{array}$ & $\begin{array}{l}\text { direct } \\
\text { death }\end{array}$ & $\begin{array}{l}\text { lost } \\
\text { f.u. }\end{array}$ & $\begin{array}{l}1 \mathrm{ysr} \\
(\%)\end{array}$ & $\begin{array}{l}2 \text { ysr } \\
(\%)\end{array}$ & $\begin{array}{l}3 \text { ysr } \\
(\%)\end{array}$ & $\begin{array}{l}4 \text { ysr } \\
(\%)\end{array}$ & $\begin{array}{l}5 y s r \\
(\%)\end{array}$ & $\begin{array}{l}5 \% \\
\text { s.d. }\end{array}$ & alive & $\begin{array}{l}\text { local } \\
\text { rec. }\end{array}$ & $\begin{array}{l}\text { peri- } \\
\text { toneal }\end{array}$ & $\begin{array}{l}\text { liver } \\
\text { rec. }\end{array}$ & $\begin{array}{l}\text { distant } \\
\text { meta. }\end{array}$ & $\mathrm{R}$ & $\begin{array}{l}\text { other } \\
\text { cancer }\end{array}$ & $\begin{array}{c}\text { other } \\
\text { disease }\end{array}$ & $\begin{array}{c}\text { un- } \\
\text { known }\end{array}$ \\
\hline T1 & 1019 & 7 & 61 & 95.4 & 93.1 & 90.1 & 88.4 & 86.5 & 2.2 & 825 & 6 & 4 & 3 & 2 & 3 & 30 & 63 & 15 \\
\hline pT2 & 239 & 3 & 26 & 70.3 & 54.7 & 47.6 & 44.1 & 40.6 & 6.7 & 81 & 23 & 28 & 25 & 2 & 13 & 3 & 25 & 10 \\
\hline pT3 & 316 & 9 & 33 & 40.8 & 20.2 & 11.8 & 9.3 & 8.0 & 3.4 & 18 & 28 & 119 & 46 & 10 & 24 & 4 & 21 & 4 \\
\hline pT4 & 210 & 4 & 19 & 31.5 & 13.6 & 8.9 & 7.0 & 7.0 & 3.9 & 11 & 33 & 77 & 19 & 4 & 26 & 1 & 13 & 3 \\
\hline Not specified & 1 & 0 & 0 & 100.0 & 100.0 & 100.0 & 100.0 & 100.0 & 0.0 & 1 & 0 & 0 & 0 & 0 & 0 & 0 & 0 & 0 \\
\hline
\end{tabular}

Table 15. pT classification by D2, D3, or D4 dissection (resected cases)

\begin{tabular}{|c|c|c|c|c|c|c|c|c|c|c|c|c|c|c|c|c|c|c|}
\hline categories & $\begin{array}{c}\text { total } \\
\text { number }\end{array}$ & $\begin{array}{l}\text { direct } \\
\text { death }\end{array}$ & $\begin{array}{l}\text { lost } \\
\text { f.u. }\end{array}$ & $\begin{array}{l}1 \text { ysr } \\
(\%)\end{array}$ & $\begin{array}{l}2 \text { ysr } \\
(\%)\end{array}$ & $\begin{array}{r}3 \text { ysr } \\
(\%)\end{array}$ & $\begin{array}{l}4 \mathrm{ysr} \\
(\%)\end{array}$ & $\begin{array}{l}5 \text { ysr } \\
(\%)\end{array}$ & $\begin{array}{l}5 \% \\
\text { s.d. }\end{array}$ & alive & $\begin{array}{l}\text { local } \\
\text { rec. }\end{array}$ & $\begin{array}{l}\text { peri- } \\
\text { toneal }\end{array}$ & $\begin{array}{l}\text { liver } \\
\text { rec. }\end{array}$ & $\begin{array}{l}\text { distant } \\
\text { meta. }\end{array}$ & $\mathrm{R}$ & $\begin{array}{l}\text { other } \\
\text { cancer }\end{array}$ & $\begin{array}{c}\text { other } \\
\text { disease }\end{array}$ & $\begin{array}{c}\text { un- } \\
\text { known }\end{array}$ \\
\hline T1 & 2852 & 8 & 175 & 97.8 & 96.2 & 94.4 & 93.3 & 91.8 & 1.0 & 2453 & 17 & 14 & 17 & 7 & 11 & 31 & 98 & 21 \\
\hline $\mathrm{T} 2$ & 1714 & 24 & 118 & 90.7 & 81.3 & 74.7 & 71.3 & 68.4 & 2.3 & 1079 & 65 & 116 & 81 & 32 & 56 & 22 & 74 & 47 \\
\hline pT3 & 1274 & 20 & 96 & 77.0 & 57.8 & 48.7 & 42.3 & 39.0 & 2.8 & 439 & 119 & 288 & 71 & 41 & 89 & 13 & 72 & 26 \\
\hline pT4 & 309 & 6 & 19 & 59.4 & 36.6 & 28.8 & 24.4 & 21.5 & 4.9 & 59 & 27 & 91 & 24 & 10 & 44 & 0 & 24 & 5 \\
\hline Not specified & 0 & 0 & 0 & - & - & - & - & - & - & 0 & 0 & 0 & 0 & 0 & 0 & 0 & 0 & 0 \\
\hline
\end{tabular}

Table 16. pT classification by curability-A resection (resected cases)

\begin{tabular}{|c|c|c|c|c|c|c|c|c|c|c|c|c|c|c|c|c|c|c|}
\hline categories & $\begin{array}{c}\text { total } \\
\text { number }\end{array}$ & $\begin{array}{l}\text { direct } \\
\text { death }\end{array}$ & $\begin{array}{l}\text { lost } \\
\text { f.u. }\end{array}$ & $\begin{array}{l}1 \text { ysr } \\
(\%)\end{array}$ & $\begin{array}{l}2 y s r \\
(\%)\end{array}$ & $\begin{array}{l}3 \text { ysr } \\
(\%)\end{array}$ & $\begin{array}{l}4 y s r \\
(\%)\end{array}$ & $\begin{array}{l}5 y s r \\
(\%)\end{array}$ & $\begin{array}{l}5 \% \\
\text { s.d. }\end{array}$ & alive & $\begin{array}{c}\text { local } \\
\text { rec. }\end{array}$ & $\begin{array}{l}\text { peri- } \\
\text { toneal }\end{array}$ & $\begin{array}{c}\text { liver } \\
\text { rec. }\end{array}$ & $\begin{array}{c}\text { distant } \\
\text { meta. }\end{array}$ & $\mathrm{R}$ & $\begin{array}{l}\text { other } \\
\text { cancer }\end{array}$ & $\begin{array}{c}\text { other } \\
\text { disease }\end{array}$ & $\begin{array}{c}\text { un- } \\
\text { known }\end{array}$ \\
\hline pT1 & 3665 & 13 & 220 & 97.6 & 96.0 & 94.1 & 92.8 & 91.4 & 0.9 & 3140 & 13 & 11 & 11 & 4 & 11 & 57 & 151 & 34 \\
\hline pT2 & 1294 & 10 & 85 & 95.6 & 90.1 & 85.9 & 82.9 & 80.3 & 2.3 & 965 & 19 & 40 & 31 & 11 & 22 & 18 & 58 & 35 \\
\hline Not specified & 0 & 0 & 0 & - & - & - & - & - & - & 0 & 0 & 0 & 0 & 0 & 0 & 0 & 0 & 0 \\
\hline
\end{tabular}

Table 17. pT classification by curability-B resection (resected cases)

\begin{tabular}{|c|c|c|c|c|c|c|c|c|c|c|c|c|c|c|c|c|c|c|}
\hline categories & $\begin{array}{c}\text { total } \\
\text { number }\end{array}$ & $\begin{array}{l}\text { direct } \\
\text { death }\end{array}$ & $\begin{array}{l}\text { lost } \\
\text { f.u. }\end{array}$ & $\begin{array}{l}1 \text { ysr } \\
(\%)\end{array}$ & $\begin{array}{l}2 y s r \\
(\%)\end{array}$ & $\begin{array}{c}3 \text { ysr } \\
(\%)\end{array}$ & $\begin{array}{l}4 y s r \\
(\%)\end{array}$ & $\begin{array}{l}5 y s r \\
(\%)\end{array}$ & $\begin{array}{l}5 \% \\
\text { s.d. }\end{array}$ & alive & $\begin{array}{c}\text { local } \\
\text { rec. }\end{array}$ & $\begin{array}{l}\text { peri- } \\
\text { toneal }\end{array}$ & $\begin{array}{c}\text { liver } \\
\text { rec. }\end{array}$ & $\begin{array}{c}\text { distant } \\
\text { meta. }\end{array}$ & $\mathrm{R}$ & $\begin{array}{l}\text { other } \\
\text { cancer }\end{array}$ & $\begin{array}{c}\text { other } \\
\text { disease }\end{array}$ & $\begin{array}{c}\text { un- } \\
\text { known }\end{array}$ \\
\hline pT1 & 175 & 1 & 14 & 93.6 & 90.1 & 85.9 & 84.6 & 80.9 & 6.1 & 129 & 3 & 4 & 5 & 3 & 3 & 4 & 7 & 2 \\
\hline pT2 & 417 & 8 & 39 & 86.7 & 70.6 & 57.7 & 53.1 & 50.1 & 5.1 & 185 & 32 & 45 & 27 & 14 & 23 & 4 & 27 & 13 \\
\hline pT3 & 984 & 15 & 80 & 85.4 & 69.1 & 60.2 & 52.7 & 48.9 & 3.3 & 428 & 77 & 173 & 29 & 29 & 61 & 14 & 55 & 23 \\
\hline pT4 & 163 & 3 & 13 & 74.4 & 55.5 & 48.0 & 42.5 & 37.6 & 7.9 & 54 & 11 & 30 & 9 & 5 & 17 & 0 & 18 & 3 \\
\hline Not specified & 0 & 0 & 0 & - & - & - & - & - & - & 0 & 0 & 0 & 0 & 0 & 0 & 0 & 0 & 0 \\
\hline
\end{tabular}

Footnote for Tables 14, 15, 27, 28, 34: Extent of LN dissection

Letter D indicates extent of lymph node (LN) dissection. Japanese $\mathrm{N}$-classification (refer to footnote on page 59) and D-classification have an anatomical base, and are completely different from the present TNM N-Classification, 5th edition. For a detailed explanation, refer to Japanese Classification of Gastric Carcinoma, 2nd English edition.

D0: No or incomplete dissection of the N1 nodes

D1: Dissection of all the N1 nodes

D2: Dissection of all the N1 and N2 nodes

D3: Dissection of all the N1, N2, and N3 nodes

Dissection of para-aortic LNs was classified as "D4" in the 12th edition. These procedures are now included in D3. D3 and D4 were called as "extended LN dissection" and D2-D4 were called as "systematic LN dissection."
Footnote for Tables 16-18: Curative potential of resection

The curative potential of gastric resection is evaluated on the following basis:

Resection A: No residual disease, with high probability of cure (see below)

Resection B: No residual disease, but not fullfilling criteria for Resection A

Resection C: Definite residual disease

Resection A implies resection satisfying all of the following conditions: T1 or T2; N0 treated by D1, or more extended dissection or N1 treated by $\mathrm{D} 2$, or more extended dissection; M0, P0, H0, CY0 and proximal and distal margins $>10 \mathrm{~mm}$.

Resection $\mathrm{A}+\mathrm{B}$ corresponds to R0 in the UICC-TNM classification and Resection C corresponds to R 1+ R2. 
Table 18. pT classification by curability-C resection (resected cases)

total direct lost 1 ysr 2 ysr 3 ysr 4 ysr 5 ysr $5 \%$ local peri- liver distant other other un-
number death f.u. (\%) (\%) (\%) (\%) (\%) s.d. alive rec. toneal rec. meta. R cancer disease known

\begin{tabular}{|c|c|c|c|c|c|c|c|c|c|c|c|c|c|c|c|c|c|c|}
\hline pT1 & 31 & 1 & 2 & 66.7 & 52.6 & 38.6 & 35.1 & 31.6 & 17.3 & 9 & 7 & 3 & 4 & 2 & 0 & 0 & 3 & 0 \\
\hline pT2 & 242 & 9 & 20 & 51.0 & 24.3 & 14.8 & 10.3 & 5.0 & 3.1 & 10 & 37 & 59 & 48 & 9 & 24 & 3 & 14 & 9 \\
\hline pT3 & 606 & 14 & 49 & 44.6 & 19.6 & 10.3 & 7.9 & 6.3 & 2.2 & 29 & 70 & 234 & 88 & 22 & 52 & 3 & 38 & 7 \\
\hline pT4 & 356 & 7 & 25 & 36.1 & 14.3 & 8.1 & 5.8 & 5.5 & 2.6 & 16 & 49 & 138 & 34 & 9 & 53 & 1 & 19 & 5 \\
\hline Not specified & 0 & 0 & 0 & - & - & - & - & - & - & 0 & 0 & 0 & 0 & 0 & 0 & 0 & 0 & 0 \\
\hline
\end{tabular}

Table 19. Macroscopic type (resected cases)

\begin{tabular}{|c|c|c|c|c|c|c|c|c|c|c|c|c|c|c|c|c|c|c|}
\hline categories & $\begin{array}{c}\text { total } \\
\text { number }\end{array}$ & $\begin{array}{l}\text { direct } \\
\text { death }\end{array}$ & $\begin{array}{l}\text { lost } \\
\text { f.u. }\end{array}$ & $\begin{array}{l}1 \text { ysr } \\
(\%)\end{array}$ & $\begin{array}{l}2 \text { ysr } \\
(\%)\end{array}$ & $\begin{array}{r}3 \text { ysr } \\
(\%)\end{array}$ & $\begin{array}{l}4 \text { ysr } \\
(\%)\end{array}$ & $\begin{array}{l}5 \text { ysr } \\
(\%)\end{array}$ & $\begin{array}{l}5 \% \\
\text { s.d. }\end{array}$ & alive & $\begin{array}{l}\text { local } \\
\text { rec. }\end{array}$ & $\begin{array}{l}\text { peri- } \\
\text { toneal }\end{array}$ & $\begin{array}{l}\text { liver } \\
\text { rec. }\end{array}$ & $\begin{array}{l}\text { distant } \\
\text { meta. }\end{array}$ & $\mathrm{R}$ & $\begin{array}{l}\text { other } \\
\text { cancer }\end{array}$ & $\begin{array}{c}\text { other } \\
\text { disease }\end{array}$ & $\begin{array}{c}\text { un- } \\
\text { known }\end{array}$ \\
\hline Type 0 (early cancer) & 4055 & 18 & 255 & 97.1 & 95.1 & 93.0 & 91.6 & 90.0 & 1.0 & 3410 & 25 & 28 & 23 & 13 & 17 & 62 & 165 & 39 \\
\hline Type 1 & 208 & 4 & 14 & 81.9 & 71.3 & 64.7 & 60.6 & 56.4 & 7.0 & 107 & 11 & 15 & 17 & 2 & 8 & 8 & 13 & 9 \\
\hline Type 2 & 1004 & 16 & 71 & 81.2 & 66.9 & 59.7 & 55.1 & 52.5 & 3.2 & 479 & 62 & 84 & 110 & 20 & 55 & 16 & 68 & 23 \\
\hline Type 3 & 1752 & 25 & 148 & 73.8 & 58.1 & 49.7 & 45.9 & 42.8 & 2.5 & 658 & 138 & 336 & 12 & 53 & 130 & 15 & 98 & 39 \\
\hline Type 4 & 556 & 14 & 39 & 56.0 & 32.7 & 24.0 & 18.7 & 16.2 & 3.3 & 78 & 62 & 240 & 13 & 16 & 51 & 2 & 31 & 10 \\
\hline Type 5 (unclassified) & 359 & 4 & 21 & 89.5 & 81.2 & 75.9 & 71.1 & 69.6 & 5.0 & 233 & 20 & 34 & 11 & 4 & 5 & 1 & 15 & 11 \\
\hline Not specified & 1 & 0 & 0 & 100.0 & 100.0 & 100.0 & 100.0 & 100.0 & 0.0 & 1 & 0 & 0 & 0 & 0 & 0 & 0 & 0 & 0 \\
\hline
\end{tabular}

Table 20. Histological type (resected cases)

\begin{tabular}{|c|c|c|c|c|c|c|c|c|c|c|c|c|c|c|c|c|c|c|}
\hline categories & $\begin{array}{c}\text { total } \\
\text { number }\end{array}$ & $\begin{array}{l}\text { direct } \\
\text { death }\end{array}$ & $\begin{array}{l}\text { lost } \\
\text { f.u. }\end{array}$ & $\begin{array}{l}1 \mathrm{ysr} \\
(\%)\end{array}$ & $\begin{array}{l}2 \text { ysr } \\
(\%)\end{array}$ & $\begin{array}{r}3 \text { ysr } \\
(\%)\end{array}$ & $\begin{array}{l}4 \text { ysr } \\
(\%)\end{array}$ & $\begin{array}{l}5 y s r \\
(\%)\end{array}$ & $\begin{array}{l}5 \% \\
\text { s.d. }\end{array}$ & alive & $\begin{array}{l}\text { local } \\
\text { rec. }\end{array}$ & $\begin{array}{l}\text { peri- } \\
\text { toneal }\end{array}$ & $\begin{array}{l}\text { liver } \\
\text { rec. }\end{array}$ & $\begin{array}{c}\text { distant } \\
\text { meta. }\end{array}$ & $\mathrm{R}$ & $\begin{array}{l}\text { other } \\
\text { cancer }\end{array}$ & $\begin{array}{c}\text { other } \\
\text { disease }\end{array}$ & $\begin{array}{c}\text { un- } \\
\text { known }\end{array}$ \\
\hline Papillary ca. & 453 & 6 & 23 & 85.0 & 72.0 & 66.9 & 65.0 & 63.4 & 4.6 & 269 & 17 & 25 & 43 & 5 & 21 & 10 & 28 & 6 \\
\hline Well differentiated & 1941 & 12 & 141 & 93.4 & 88.6 & 85.6 & 83.4 & 81.1 & 1.8 & 1448 & 40 & 53 & 45 & 7 & 28 & 39 & 105 & 23 \\
\hline Mod. differentiateds & 1889 & 21 & 127 & 87.3 & 78.7 & 72.8 & 69.7 & 67.8 & 2.2 & 1176 & 66 & 111 & 106 & 30 & 65 & 29 & 119 & 39 \\
\hline Poorly ca. solid & 1297 & 15 & 70 & 80.1 & 69.9 & 65.1 & 61.7 & 59.0 & 2.8 & 713 & 91 & 172 & 59 & 28 & 45 & 10 & 61 & 33 \\
\hline Poorly, non-solid & 1297 & 19 & 107 & 80.0 & 67.7 & 61.1 & 57.9 & 55.5 & 2.9 & 645 & 57 & 276 & 19 & 26 & 74 & 9 & 50 & 15 \\
\hline Signet ring ca. & 825 & 4 & 59 & 91.1 & 87.2 & 84.3 & 82.5 & 81.2 & 2.8 & 616 & 23 & 64 & 3 & 8 & 13 & 5 & 20 & 10 \\
\hline Mucinous ca. & 192 & 3 & 20 & 77.3 & 64.2 & 56.2 & 51.5 & 49.7 & 7.5 & 82 & 21 & 31 & 6 & 3 & 17 & 1 & 4 & 4 \\
\hline Other types & 41 & 1 & 1 & 72.8 & 60.3 & 52.7 & 47.7 & 42.7 & 15.6 & 17 & 3 & 5 & 5 & 1 & 3 & 1 & 3 & 1 \\
\hline
\end{tabular}

Table 21. Lauren's histological type (resected cases)

\begin{tabular}{|c|c|c|c|c|c|c|c|c|c|c|c|c|c|c|c|c|c|c|}
\hline categories & $\begin{array}{c}\text { total } \\
\text { number }\end{array}$ & $\begin{array}{l}\text { direct } \\
\text { death }\end{array}$ & $\begin{array}{l}\text { lost } \\
\text { f.u. }\end{array}$ & $\begin{array}{l}1 \text { ysr } \\
(\%)\end{array}$ & $\begin{array}{l}2 y s r \\
(\%)\end{array}$ & $\begin{array}{r}3 \text { ysr } \\
(\%)\end{array}$ & $\begin{array}{l}4 y s r \\
(\%)\end{array}$ & $\begin{array}{l}5 \text { ysr } \\
(\%)\end{array}$ & $\begin{array}{l}5 \% \\
\text { s.d. }\end{array}$ & alive & $\begin{array}{c}\text { local } \\
\text { rec. }\end{array}$ & $\begin{array}{l}\text { peri- } \\
\text { toneal }\end{array}$ & $\begin{array}{l}\text { liver } \\
\text { rec. }\end{array}$ & $\begin{array}{l}\text { distant } \\
\text { meta. }\end{array}$ & $\mathrm{R}$ & $\begin{array}{l}\text { other } \\
\text { cancer }\end{array}$ & $\begin{array}{l}\text { other } \\
\text { disease }\end{array}$ & $\begin{array}{c}\text { un- } \\
\text { known }\end{array}$ \\
\hline Intestinal type & 4283 & 39 & 291 & 89.8 & 82.4 & 78.0 & 75.4 & 73.3 & 1.4 & 2893 & 123 & 189 & 194 & 42 & 114 & 78 & 252 & 68 \\
\hline Diffuse type & 3611 & 41 & 256 & 82.4 & 72.8 & 67.6 & 64.6 & 62.4 & 1.7 & 2056 & 192 & 543 & 87 & 65 & 149 & 25 & 135 & 62 \\
\hline Other type & 40 & 1 & 1 & 72.2 & 59.3 & 51.5 & 46.4 & 41.2 & 15.8 & 16 & 3 & 5 & 5 & 1 & 3 & 1 & 3 & 1 \\
\hline Not specified & 1 & 0 & 0 & 100.0 & 100.0 & 100.0 & 100.0 & 100.0 & 0.0 & 1 & 0 & 0 & 0 & 0 & 0 & 0 & 0 & 0 \\
\hline
\end{tabular}

Footnote for Table 19: Macroscopic type

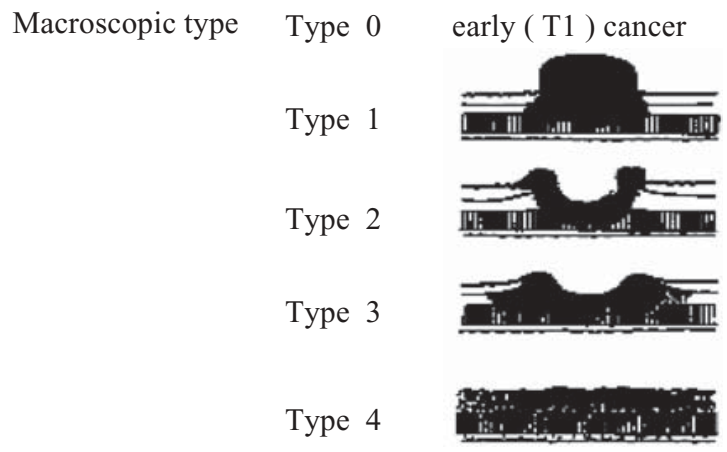

Footnote for Table 20: Histological type

The histological classification should be based on the predominant pattern of the tumor. Following are the classifications of common histological types of gastric carcinoma.

$\begin{array}{ll}\begin{array}{l}\text { Papillary adenocarcinoma } \\ \text { Tubular adenocarcinoma }\end{array} & \begin{array}{l}\text { Well-differentiated type } \\ \text { Moderately differentiated type } \\ \text { Solid type }\end{array} \\ \text { Noorly differentiated adenoca } & \begin{array}{l}\text { Non-solid type } \\ \text { Signet-ring cell carcinoma }\end{array}\end{array}$

Mucinous adenocarcinoma
Well-differentiated type Moderately differentiated type Non-solid type 
Table 22. Cancer-stroma relationship (resected cases)

\begin{tabular}{|c|c|c|c|c|c|c|c|c|c|c|c|c|c|c|c|c|c|c|}
\hline categories & $\begin{array}{c}\text { total } \\
\text { number }\end{array}$ & $\begin{array}{l}\text { direct } \\
\text { death }\end{array}$ & $\begin{array}{l}\text { lost } \\
\text { f.u. }\end{array}$ & $\begin{array}{l}1 \text { ysr } \\
(\%)\end{array}$ & $\begin{array}{l}2 \text { ysr } \\
(\%)\end{array}$ & $\begin{array}{r}3 \text { ysr } \\
(\%)\end{array}$ & $\begin{array}{l}4 \text { ysr } \\
(\%)\end{array}$ & $\begin{array}{l}5 \text { ysr } \\
(\%)\end{array}$ & $\begin{array}{l}5 \% \\
\text { s.d. }\end{array}$ & alive & $\begin{array}{l}\text { local } \\
\text { rec. }\end{array}$ & $\begin{array}{l}\text { peri- } \\
\text { toneal }\end{array}$ & $\begin{array}{l}\text { liver } \\
\text { rec. }\end{array}$ & $\begin{array}{l}\text { distant } \\
\text { meta. }\end{array}$ & $\mathrm{R}$ & $\begin{array}{l}\text { other } \\
\text { cancer }\end{array}$ & $\begin{array}{c}\text { other } \\
\text { disease }\end{array}$ & $\begin{array}{c}\text { un- } \\
\text { known }\end{array}$ \\
\hline Medullary & 1115 & 5 & 84 & 89.5 & 81.4 & 78.1 & 75.3 & 72.4 & 2.8 & 738 & 31 & 56 & 56 & 13 & 48 & 19 & 46 & 19 \\
\hline Intermediate & 1594 & 17 & 139 & 85.3 & 76.3 & 71.1 & 68.3 & 65.7 & 2.4 & 935 & 73 & 119 & 82 & 24 & 71 & 22 & 88 & 24 \\
\hline Scirrhous & 891 & 11 & 87 & 76.9 & 62.2 & 54.3 & 50.5 & 47.2 & 3.5 & 362 & 40 & 242 & 23 & 20 & 63 & 3 & 31 & 9 \\
\hline Not specified & 4335 & 48 & 238 & 87.9 & 80.9 & 76.5 & 73.8 & 72.2 & 1.4 & 2931 & 174 & 320 & 125 & 51 & 84 & 60 & 225 & 79 \\
\hline
\end{tabular}

Table 23. Infiltration pattern (INF) (resected cases)

\begin{tabular}{|c|c|c|c|c|c|c|c|c|c|c|c|c|c|c|c|c|c|c|}
\hline categories & $\begin{array}{l}\text { total } \\
\text { number }\end{array}$ & $\begin{array}{l}\text { direct } \\
\text { death }\end{array}$ & $\begin{array}{l}\text { lost } \\
\text { f.u. }\end{array}$ & $\begin{array}{l}1 \text { ysr } \\
(\%)\end{array}$ & $\begin{array}{l}2 \mathrm{ysr} \\
(\%)\end{array}$ & $\begin{array}{l}3 \text { ysr } \\
(\%)\end{array}$ & $\begin{array}{l}4 \text { ysr } \\
(\%)\end{array}$ & $\begin{array}{l}5 y s r \\
(\%)\end{array}$ & $\begin{array}{l}5 \% \\
\text { s.d. }\end{array}$ & alive & $\begin{array}{l}\text { local } \\
\text { rec. }\end{array}$ & $\begin{array}{l}\text { peri- } \\
\text { toneal }\end{array}$ & $\begin{array}{l}\text { liver } \\
\text { rec. }\end{array}$ & $\begin{array}{l}\text { distant } \\
\text { meta. }\end{array}$ & $\mathrm{R}$ & $\begin{array}{l}\text { other } \\
\text { cancer }\end{array}$ & $\begin{array}{c}\text { other } \\
\text { disease }\end{array}$ & $\begin{array}{c}\text { un- } \\
\text { known }\end{array}$ \\
\hline INF $\alpha$ & 1678 & 10 & 102 & 94.6 & 90.9 & 88.5 & 86.6 & 84.6 & 1.8 & 1327 & 19 & 33 & 35 & 10 & 17 & 28 & 78 & 19 \\
\hline INF $\beta$ & 2873 & 31 & 189 & 86.8 & 78.1 & 73.0 & 70.3 & 68.3 & 1.8 & 1805 & 126 & 177 & 169 & 40 & 94 & 47 & 143 & 52 \\
\hline INF $\gamma$ & 1983 & 30 & 161 & 75.5 & 61.5 & 54.4 & 50.4 & 47.5 & 2.3 & 836 & 143 & 450 & 57 & 46 & 131 & 5 & 92 & 32 \\
\hline Not specified & 1401 & 10 & 96 & 90.9 & 84.9 & 81.1 & 78.7 & 77.2 & 2.3 & 998 & 30 & 77 & 25 & 12 & 24 & 24 & 77 & 28 \\
\hline
\end{tabular}

Table 24. Lymphatic invasion (ly) (resected cases)

\begin{tabular}{|c|c|c|c|c|c|c|c|c|c|c|c|c|c|c|c|c|c|c|}
\hline categories & $\begin{array}{c}\text { total } \\
\text { number }\end{array}$ & $\begin{array}{l}\text { direct } \\
\text { death }\end{array}$ & $\begin{array}{l}\text { lost } \\
\text { f.u. }\end{array}$ & $\begin{array}{l}1 \mathrm{ysr} \\
(\%)\end{array}$ & $\begin{array}{l}2 \text { ysr } \\
(\%)\end{array}$ & $\begin{array}{l}3 \text { ysr } \\
(\%)\end{array}$ & $\begin{array}{l}4 \text { ysr } \\
(\%)\end{array}$ & $\begin{array}{l}5 \text { ysr } \\
(\%)\end{array}$ & $\begin{array}{l}5 \% \\
\text { s.d. }\end{array}$ & alive & $\begin{array}{c}\text { local } \\
\text { rec. }\end{array}$ & $\begin{array}{l}\text { peri- } \\
\text { toneal }\end{array}$ & $\begin{array}{l}\text { liver } \\
\text { rec. }\end{array}$ & $\begin{array}{c}\text { distant } \\
\text { meta. }\end{array}$ & $\mathrm{R}$ & $\begin{array}{l}\text { other } \\
\text { cancer }\end{array}$ & $\begin{array}{c}\text { other } \\
\text { disease }\end{array}$ & $\begin{array}{c}\text { un- } \\
\text { known }\end{array}$ \\
\hline ly 0 & 3511 & 18 & 215 & 96.6 & 94.1 & 91.8 & 90.3 & 88.7 & 1.1 & 2915 & 18 & 72 & 18 & 10 & 23 & 48 & 129 & 45 \\
\hline ly 1 & 1669 & 15 & 122 & 88.9 & 81.0 & 75.1 & 71.6 & 69.3 & 2.3 & 1057 & 53 & 141 & 59 & 21 & 39 & 27 & 106 & 29 \\
\hline ly 2 & 1400 & 20 & 114 & 77.8 & 61.4 & 53.8 & 49.7 & 46.5 & 2.8 & 576 & 105 & 244 & 110 & 35 & 77 & 18 & 77 & 24 \\
\hline ly 3 & 1070 & 25 & 82 & 59.6 & 40.0 & 32.0 & 27.5 & 24.7 & 2.8 & 230 & 131 & 263 & 95 & 41 & 114 & 5 & 59 & 25 \\
\hline Not specified & 285 & 3 & 15 & 86.8 & 79.2 & 74.8 & 72.2 & 70.3 & 5.5 & 188 & 11 & 17 & 4 & 1 & 13 & 6 & 19 & 8 \\
\hline
\end{tabular}

Table 25. Venous invasion (v) (resected cases)

\begin{tabular}{|c|c|c|c|c|c|c|c|c|c|c|c|c|c|c|c|c|c|c|}
\hline categories & $\begin{array}{c}\text { total } \\
\text { number }\end{array}$ & $\begin{array}{l}\text { direct } \\
\text { death }\end{array}$ & $\begin{array}{l}\text { lost } \\
\text { f.u. }\end{array}$ & $\begin{array}{l}1 \text { ysr } \\
(\%)\end{array}$ & $\begin{array}{l}2 y s r \\
(\%)\end{array}$ & $\begin{array}{r}3 \text { ysr } \\
(\%)\end{array}$ & $\begin{array}{l}4 \text { ysr } \\
(\%)\end{array}$ & $\begin{array}{l}5 y s r \\
(\%)\end{array}$ & $\begin{array}{l}5 \% \\
\text { s.d. }\end{array}$ & alive & $\begin{array}{l}\text { local } \\
\text { rec. }\end{array}$ & $\begin{array}{l}\text { peri- } \\
\text { toneal }\end{array}$ & $\begin{array}{l}\text { liver } \\
\text { rec. }\end{array}$ & $\begin{array}{c}\text { distant } \\
\text { meta. }\end{array}$ & $\mathrm{R}$ & $\begin{array}{c}\text { other } \\
\text { cancer }\end{array}$ & $\begin{array}{c}\text { other } \\
\text { disease }\end{array}$ & $\begin{array}{c}\text { un- } \\
\text { known }\end{array}$ \\
\hline v 0 & 5210 & 36 & 342 & 93.0 & 88.0 & 84.3 & 82.1 & 80.1 & 1.1 & 3872 & 105 & 291 & 67 & 49 & 83 & 76 & 218 & 71 \\
\hline v 1 & 1440 & 18 & 120 & 78.2 & 63.6 & 55.9 & 51.6 & 49.2 & 2.7 & 626 & 111 & 221 & 83 & 35 & 82 & 17 & 92 & 35 \\
\hline v 2 & 663 & 11 & 45 & 65.3 & 48.5 & 41.7 & 37.2 & 34.5 & 3.8 & 204 & 70 & 120 & 84 & 15 & 54 & 4 & 42 & 14 \\
\hline v 3 & 302 & 12 & 23 & 58.0 & 35.0 & 28.4 & 25.0 & 23.5 & 5.1 & 60 & 21 & 79 & 48 & 8 & 33 & 1 & 14 & 3 \\
\hline Not specified & 320 & 4 & 18 & 85.4 & 78.3 & 73.7 & 71.4 & 68.4 & 5.3 & 204 & 11 & 26 & 4 & 1 & 14 & 6 & 24 & 8 \\
\hline
\end{tabular}

Footnote for Tables 22, 23:

Cancer-stroma relationship

$\begin{array}{ll}\text { Medullary type: } & \text { Stroma is scanty } \\ \text { Scirrhous type: } & \text { Stroma is abundant } \\ \text { Intermediate type: } & \text { Quantity of stroma is intermediate }\end{array}$

Pattern of tumor infiltration into the surrounding tissue

INF $\alpha$ : The tumor shows expanding growth and a distinct border with the surrounding tissue

INF $\beta$ : This category is between INF $\alpha$ and INF $\gamma$

INF $\gamma$ : The tumor shows infiltrating growth and an indistinct border with the surrounding tissue
Footnote for Tables 24, 25:

Lymphatic invasion

ly 0: No lymphatic invasion

ly 1: Minimal lymphatic invasion

ly 2: Moderate lymphatic invasion

ly 3: Marked lymphatic invasion

Venous invasion

v 0: No venous invasion

v 1: Minimal venous invasion

v 2: Moderate venous invasion

v 3: Severe venous invasion 
Table 26. Lymph node metastasis $(\mathrm{pN})$ (resected cases)

\begin{tabular}{|c|c|c|c|c|c|c|c|c|c|c|c|c|c|c|c|c|c|c|}
\hline categories & $\begin{array}{c}\text { total } \\
\text { number }\end{array}$ & $\begin{array}{l}\text { direct } \\
\text { death }\end{array}$ & $\begin{array}{l}\text { lost } \\
\text { f.u. }\end{array}$ & $\begin{array}{l}1 \text { ysr } \\
(\%)\end{array}$ & $\begin{array}{l}2 \text { ysr } \\
(\%)\end{array}$ & $\begin{array}{r}3 \text { ysr } \\
(\%)\end{array}$ & $\begin{array}{l}4 \mathrm{ysr} \\
(\%)\end{array}$ & $\begin{array}{l}5 y s r \\
(\%)\end{array}$ & $\begin{array}{l}5 \% \\
\text { s.d. }\end{array}$ & alive & $\begin{array}{l}\text { local } \\
\text { rec. }\end{array}$ & $\begin{array}{l}\text { peri- } \\
\text { toneal }\end{array}$ & $\begin{array}{l}\text { liver } \\
\text { rec. }\end{array}$ & $\begin{array}{l}\text { distant } \\
\text { meta. }\end{array}$ & $\mathrm{R}$ & $\begin{array}{l}\text { other } \\
\text { cancer }\end{array}$ & $\begin{array}{l}\text { other } \\
\text { disease }\end{array}$ & $\begin{array}{c}\text { un- } \\
\text { knowr }\end{array}$ \\
\hline pN0 & 4700 & 23 & 292 & 96.5 & 93.9 & 91.6 & 89.8 & 87.9 & 1.0 & 3860 & 30 & 85 & 29 & 10 & 37 & 64 & 209 & 61 \\
\hline N1 & 1395 & 20 & 114 & 84.7 & 72.3 & 64.2 & 60.2 & 57.7 & 2.7 & 725 & 57 & 173 & 97 & 29 & 58 & 23 & 70 & 33 \\
\hline $\mathrm{N} 2$ & 1145 & 18 & 93 & 70.3 & 51.7 & 41.8 & 36.2 & 32.6 & 2.9 & 324 & 105 & 279 & 93 & 38 & 86 & 15 & 72 & 22 \\
\hline $\mathrm{pN} 3$ & 333 & 12 & 20 & 53.1 & 27.3 & 16.8 & 12.8 & 11.4 & 3.6 & 34 & 39 & 103 & 37 & 18 & 43 & 1 & 18 & 8 \\
\hline pN4 & 356 & 8 & 28 & 41.0 & 15.5 & 9.9 & 8.9 & 7.8 & 3.1 & 22 & 87 & 97 & 30 & 13 & 42 & 1 & 21 & 7 \\
\hline Not specified & 2 & 0 & 1 & 100.0 & 100.0 & 100.0 & 100.0 & 100.0 & 0.0 & 1 & 0 & 0 & 0 & 0 & 0 & 0 & 0 & 0 \\
\hline$p N 3+p N 4$ & 689 & 20 & 48 & 46.9 & 21.3 & 13.3 & 10.8 & 9.6 & 2.4 & 56 & 126 & 200 & 67 & 31 & 85 & 2 & 39 & 15 \\
\hline
\end{tabular}

Table 27. Lymph node metastasis $(\mathrm{pN})$ by $\mathrm{D} 0$ or D1 dissection (resected cases)

\begin{tabular}{|c|c|c|c|c|c|c|c|c|c|c|c|c|c|c|c|c|c|c|}
\hline categories & $\begin{array}{c}\text { total } \\
\text { number }\end{array}$ & $\begin{array}{l}\text { direct } \\
\text { death }\end{array}$ & $\begin{array}{l}\text { lost } \\
\text { f.u. }\end{array}$ & $\begin{array}{l}1 \mathrm{ysr} \\
(\%)\end{array}$ & $\begin{array}{l}2 \mathrm{ysr} \\
(\%)\end{array}$ & $\begin{array}{l}3 \text { ysr } \\
(\%)\end{array}$ & $\begin{array}{l}4 y s r \\
(\%)\end{array}$ & $\begin{array}{l}5 \text { ysr } \\
(\%)\end{array}$ & $\begin{array}{l}5 \% \\
\text { s.d. }\end{array}$ & alive & $\begin{array}{c}\text { local } \\
\text { rec. }\end{array}$ & $\begin{array}{l}\text { peri- } \\
\text { toneal }\end{array}$ & $\begin{array}{c}\text { liver } \\
\text { rec. }\end{array}$ & $\begin{array}{c}\text { distant } \\
\text { meta. }\end{array}$ & $\mathrm{R}$ & $\begin{array}{l}\text { other } \\
\text { cancer }\end{array}$ & $\begin{array}{c}\text { other } \\
\text { disease }\end{array}$ & $\begin{array}{c}\text { un- } \\
\text { known }\end{array}$ \\
\hline pN0 & 1108 & 8 & 74 & 94.7 & 91.4 & 87.9 & 85.8 & 83.7 & 2.3 & 860 & 9 & 17 & 10 & 3 & 5 & 27 & 76 & 19 \\
\hline $\mathrm{pN} 1$ & 228 & 4 & 24 & 57.0 & 42.9 & 35.7 & 34.7 & 33.1 & 6.6 & 63 & 14 & 57 & 25 & 3 & 12 & 6 & 15 & 5 \\
\hline $\mathrm{pN} 2$ & 187 & 6 & 19 & 34.3 & 18.9 & 10.3 & 6.6 & 4.0 & 3.4 & 5 & 17 & 73 & 23 & 6 & 14 & 4 & 17 & 3 \\
\hline $\mathrm{pN} 3$ & 105 & 3 & 7 & 36.9 & 10.9 & 6.5 & 3.3 & 3.3 & 3.7 & 3 & 13 & 36 & 14 & 4 & 18 & 0 & 6 & 1 \\
\hline $\mathrm{pN} 4$ & 156 & 2 & 15 & 34.4 & 10.0 & 5.2 & 4.2 & 4.2 & 3.6 & 4 & 37 & 45 & 21 & 2 & 17 & 1 & 8 & 4 \\
\hline Not specified & 1 & 0 & 0 & 100.0 & 100.0 & 100.0 & 100.0 & 100.0 & 0.0 & 1 & 0 & 0 & 0 & 0 & 0 & 0 & 0 & 0 \\
\hline$p N 3+p N 4$ & 261 & 5 & 22 & 35.5 & 10.3 & 5.7 & 3.7 & 3.7 & 2.6 & 7 & 50 & 81 & 35 & 6 & 35 & 1 & 14 & 5 \\
\hline
\end{tabular}

Table 28. Lymph node metastasis $(\mathrm{pN})$ by D2, D3, or D4 dissection (resected cases)

\begin{tabular}{|c|c|c|c|c|c|c|c|c|c|c|c|c|c|c|c|c|c|c|}
\hline categories & $\begin{array}{c}\text { total } \\
\text { number }\end{array}$ & $\begin{array}{l}\text { direct } \\
\text { death }\end{array}$ & $\begin{array}{l}\text { lost } \\
\text { f.u. }\end{array}$ & $\begin{array}{l}1 \mathrm{ysr} \\
(\%)\end{array}$ & $\begin{array}{l}2 \text { ysr } \\
(\%)\end{array}$ & $\begin{array}{r}3 \text { ysr } \\
(\%)\end{array}$ & $\begin{array}{l}4 \mathrm{ysr} \\
(\%)\end{array}$ & $\begin{array}{l}5 y s r \\
(\%)\end{array}$ & $\begin{array}{l}5 \% \\
\text { s.d. }\end{array}$ & alive & $\begin{array}{l}\text { local } \\
\text { rec. }\end{array}$ & $\begin{array}{l}\text { peri- } \\
\text { toneal }\end{array}$ & $\begin{array}{l}\text { liver } \\
\text { rec. }\end{array}$ & $\begin{array}{c}\text { distant } \\
\text { meta. }\end{array}$ & $\mathrm{R}$ & $\begin{array}{l}\text { other } \\
\text { cancer }\end{array}$ & $\begin{array}{c}\text { other } \\
\text { disease }\end{array}$ & $\begin{array}{c}\text { un- } \\
\text { known }\end{array}$ \\
\hline pNO & 3592 & 15 & 218 & 97.0 & 94.6 & 92.8 & 91.0 & 89.2 & 1.1 & 3000 & 21 & 68 & 19 & 7 & 32 & 37 & 133 & 42 \\
\hline pN1 & 1171 & 16 & 90 & 89.9 & 77.8 & 69.5 & 65.0 & 62.3 & 2.9 & 662 & 43 & 116 & 72 & 26 & 46 & 17 & 55 & 28 \\
\hline $\mathrm{pN} 2$ & 958 & 12 & 74 & 77.2 & 57.9 & 47.6 & 41.6 & 37.7 & 3.2 & 319 & 88 & 206 & 70 & 32 & 72 & 11 & 55 & 19 \\
\hline $\mathrm{pN} 3$ & 228 & 9 & 13 & 60.4 & 34.6 & 21.3 & 17.0 & 15.1 & 4.9 & 31 & 26 & 67 & 23 & 14 & 25 & 1 & 12 & 7 \\
\hline pN4 & 200 & 6 & 13 & 46.0 & 19.6 & 13.4 & 12.3 & 10.5 & 4.6 & 18 & 50 & 52 & 9 & 11 & 25 & 0 & 13 & 3 \\
\hline Not specified & 0 & 0 & 0 & - & - & - & - & - & - & 0 & 0 & 0 & 0 & 0 & 0 & 0 & 0 & 0 \\
\hline$p N 3+p N 4$ & 428 & 15 & 26 & 53.7 & 27.6 & 17.6 & 14.8 & 12.9 & 3.4 & 49 & 76 & 119 & 32 & 25 & 50 & 1 & 25 & 10 \\
\hline
\end{tabular}

Footnote for Tables 26-31: Regional LN stations and N classification by tumor location

The regional LNs are classified into four compartments depending upon the location of the primary tumor. The extent of LN metastasis $(\mathrm{N})$ is classified into the 5 categories $(\mathrm{N} 0-\mathrm{N} 4)$ in the 11th Japanese edition. Metastasis to para-aortic LNs were included in N3 in the 2nd English edition.
N0: $\quad$ No evidence of LN metastasis

N1: $\quad$ Metastasis to N1 nodes, but not to N2, N3, or N4

N2: $\quad$ Metastasis to N2 nodes, but not to N3 or N4

N3: Metastasis to N3 nodes, but not to N4

N4: $\quad$ Metastasis to N4 nodes

\begin{tabular}{|c|c|c|c|c|c|c|c|c|c|c|c|}
\hline \multicolumn{2}{|r|}{ Location } & $\begin{array}{l}\text { LMU } \\
\text { MUL } \\
\text { MLU } \\
\text { UML }\end{array}$ & $\begin{array}{c}\mathrm{L} \\
\mathrm{LM}\end{array}$ & $\begin{array}{l}\text { M } \\
\text { ML } \\
\text { MU }\end{array}$ & $\begin{array}{c}\mathrm{U} \\
\mathrm{UM}\end{array}$ & $\begin{array}{l}\text { No. } 7 \\
\text { No. } 8 \mathrm{a} \\
\text { No. } 8 \mathrm{p} \\
\text { No. } 9\end{array}$ & $\begin{array}{l}\text { rt paracardial } \\
\text { lt paracardial } \\
\text { lesser curvature } \\
\text { lt gastroepiploic }\end{array}$ & $\begin{array}{l}2 \\
2 \\
3 \\
2\end{array}$ & $\begin{array}{l}2 \\
2 \\
3 \\
2\end{array}$ & $\begin{array}{l}2 \\
2 \\
3 \\
2\end{array}$ & $\begin{array}{l}2 \\
2 \\
3 \\
2\end{array}$ \\
\hline & & & Liv & & UN & $\begin{array}{l}\text { No. } 9 \\
\text { No. } 10\end{array}$ & It gastroepiploic & 2 & 2 & 2 & 2 \\
\hline No. 1 & rt paracardial & 1 & 2 & 1 & 1 & No. 11 & suprapyloric & 2 & 3 & 2 & 2 \\
\hline No. 2 & lt paracardial & 1 & 3 & 2 & 1 & No. 12 & infrapyloric & 3 & 3 & 3 & 3 \\
\hline No. 3 & lesser curvature & 1 & 1 & 1 & 1 & No. 13 & retropancreatic & 3 & 3 & 3 & 3 \\
\hline No. $4 \mathrm{~s}$ & It gastroepiploic & 1 & 1 & 1 & 1 & No. $14 \mathrm{v}$ & sup mesenteric $\mathrm{v}$. & 3 & 3 & 3 & 3 \\
\hline No. $4 d$ & rt gastroepiploic & 1 & 1 & 1 & 2 & No. $14 \mathrm{a}$ & sup mesenteric a. & 4 & 4 & 4 & 4 \\
\hline No. 5 & suprapyloric & 1 & 1 & 1 & 2 & No. 15 & middle colic & 4 & 4 & 4 & 4 \\
\hline No. 6 & infrapyloric & 1 & 1 & 1 & 2 & No. 16 & para-aortic & 4 & 4 & 4 & 4 \\
\hline
\end{tabular}

From the 11th Japanese edition. The 2nd English edition differs in several points.

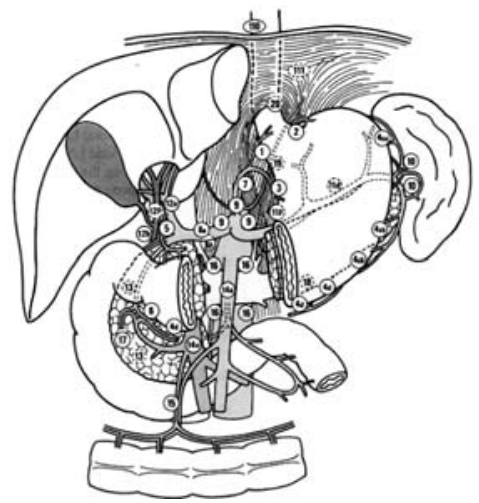




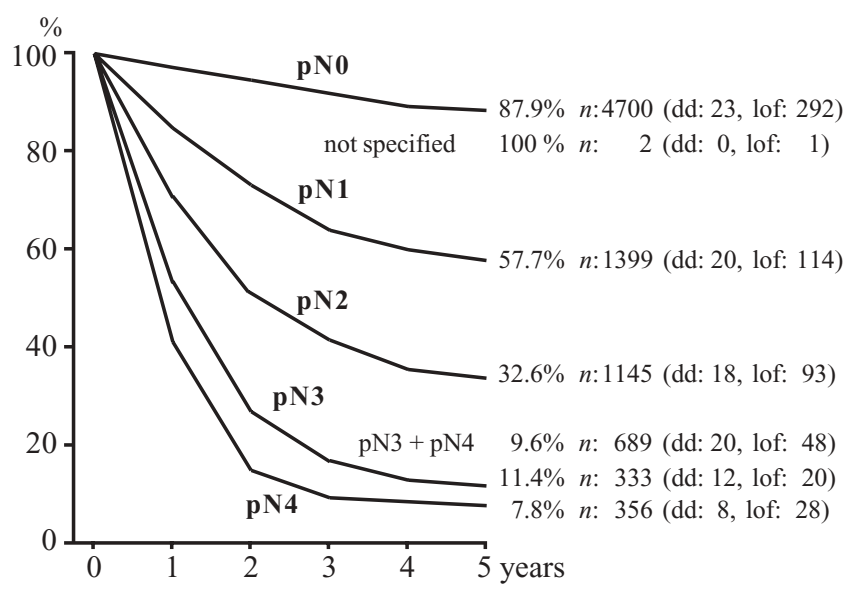

Fig. 4. Lymph node metastasis $(\mathrm{pN})$

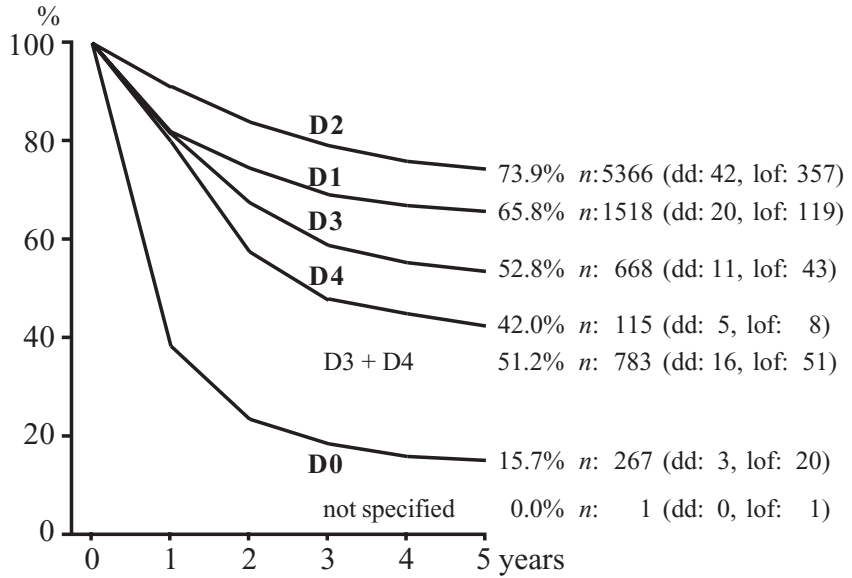

Fig. 5. Lymph node dissection (D)

Table 29. Lymph node metastasis $(\mathrm{pN})$ by curability-A resection (resected cases)

\begin{tabular}{|c|c|c|c|c|c|c|c|c|c|c|c|c|c|c|c|c|c|c|}
\hline categories & $\begin{array}{c}\text { total } \\
\text { number }\end{array}$ & $\begin{array}{l}\text { direct } \\
\text { death }\end{array}$ & $\begin{array}{l}\text { lost } \\
\text { f.u. }\end{array}$ & $\begin{array}{l}1 \mathrm{ysr} \\
(\%)\end{array}$ & $\begin{array}{l}2 \text { ysr } \\
(\%)\end{array}$ & $\begin{array}{l}3 \text { ysr } \\
(\%)\end{array}$ & $\begin{array}{l}4 \text { ysr } \\
(\%)\end{array}$ & $\begin{array}{l}5 y s r \\
(\%)\end{array}$ & $\begin{array}{l}5 \% \\
\text { s.d. }\end{array}$ & alive & $\begin{array}{l}\text { local } \\
\text { rec. }\end{array}$ & $\begin{array}{l}\text { peri- } \\
\text { toneal }\end{array}$ & $\begin{array}{l}\text { liver } \\
\text { rec. }\end{array}$ & $\begin{array}{l}\text { distant } \\
\text { meta. }\end{array}$ & $\mathrm{R}$ & $\begin{array}{l}\text { other } \\
\text { cancer }\end{array}$ & $\begin{array}{c}\text { other } \\
\text { disease }\end{array}$ & $\begin{array}{c}\text { un- } \\
\text { known }\end{array}$ \\
\hline $\mathrm{pN} 0$ & 4296 & 17 & 259 & 97.2 & 95.4 & 93.5 & 91.9 & 90.4 & 0.9 & 3639 & 17 & 31 & 16 & 3 & 20 & 63 & 177 & 54 \\
\hline pN1 & 663 & 6 & 46 & 96.0 & 88.4 & 82.0 & 78.9 & 76.1 & 3.4 & 466 & 15 & 20 & 26 & 12 & 13 & 12 & 32 & 15 \\
\hline Not specified & 0 & 0 & 0 & - & - & - & - & - & - & 0 & 0 & 0 & 0 & 0 & 0 & 0 & 0 & 0 \\
\hline
\end{tabular}

Table 30. Lymph node metastasis $(\mathrm{pN})$ by curability-B resection (resected cases)

\begin{tabular}{|c|c|c|c|c|c|c|c|c|c|c|c|c|c|c|c|c|c|c|}
\hline categories & $\begin{array}{c}\text { total } \\
\text { number }\end{array}$ & $\begin{array}{l}\text { direct } \\
\text { death }\end{array}$ & $\begin{array}{l}\text { lost } \\
\text { f.u. }\end{array}$ & $\begin{array}{l}1 \mathrm{ysr} \\
(\%)\end{array}$ & $\begin{array}{l}2 \mathrm{ysr} \\
(\%)\end{array}$ & $\begin{array}{r}3 \text { ysr } \\
(\%)\end{array}$ & $\begin{array}{l}4 y s r \\
(\%)\end{array}$ & $\begin{array}{l}5 \text { ysr } \\
(\%)\end{array}$ & $\begin{array}{l}5 \% \\
\text { s.d. }\end{array}$ & alive & $\begin{array}{l}\text { local } \\
\text { rec. }\end{array}$ & $\begin{array}{l}\text { peri- } \\
\text { toneal }\end{array}$ & $\begin{array}{l}\text { liver } \\
\text { rec. }\end{array}$ & $\begin{array}{c}\text { distant } \\
\text { meta. }\end{array}$ & $\mathrm{R}$ & $\begin{array}{l}\text { other } \\
\text { cancer }\end{array}$ & $\begin{array}{l}\text { other } \\
\text { disease }\end{array}$ & $\begin{array}{c}\text { un- } \\
\text { known }\end{array}$ \\
\hline pN0 & 333 & 4 & 27 & 93.6 & 84.9 & 80.2 & 74.7 & 69.7 & 5.2 & 210 & 10 & 30 & 3 & 3 & 13 & 1 & 25 & 7 \\
\hline pN1 & 513 & 9 & 48 & 86.4 & 72.4 & 62.4 & 57.0 & 54.4 & 4.6 & 246 & 26 & 68 & 24 & 16 & 26 & 10 & 27 & 13 \\
\hline $\mathrm{pN} 2$ & 793 & 11 & 64 & 82.6 & 65.0 & 54.8 & 47.9 & 43.8 & 3.7 & 309 & 70 & 134 & 40 & 27 & 61 & 11 & 48 & 18 \\
\hline $\mathrm{pN} 3$ & 71 & 1 & 7 & 81.0 & 56.3 & 39.1 & 34.3 & 34.3 & 11.8 & 21 & 9 & 14 & 2 & 4 & 4 & 0 & 6 & 3 \\
\hline pN4 & 29 & 2 & 0 & 65.5 & 44.8 & 41.4 & 37.9 & 34.5 & 17.7 & 10 & 8 & 6 & 1 & 1 & 0 & 0 & 1 & 0 \\
\hline Not specified & 0 & 0 & 0 & - & - & - & - & - & - & 0 & 0 & 0 & 0 & 0 & 0 & 0 & 0 & 0 \\
\hline$p N 3+p N 4$ & 100 & 3 & 7 & 76.4 & 52.9 & 39.9 & 35.6 & 34.5 & 9.8 & 31 & 17 & 20 & 3 & 5 & 4 & 0 & 7 & 3 \\
\hline
\end{tabular}

Table 31. Lymph node metastasis $(\mathrm{pN})$ by curability-C resection (resected cases)

\begin{tabular}{|c|c|c|c|c|c|c|c|c|c|c|c|c|c|c|c|c|c|c|}
\hline categories & $\begin{array}{c}\text { total } \\
\text { number }\end{array}$ & $\begin{array}{l}\text { direct } \\
\text { death }\end{array}$ & $\begin{array}{l}\text { lost } \\
\text { f.u. }\end{array}$ & $\begin{array}{l}1 \mathrm{ysr} \\
(\%)\end{array}$ & $\begin{array}{l}2 \text { ysr } \\
(\%)\end{array}$ & $\begin{array}{l}3 \text { ysr } \\
(\%)\end{array}$ & $\begin{array}{l}4 \text { ysr } \\
(\%)\end{array}$ & $\begin{array}{l}5 y s r \\
(\%)\end{array}$ & $\begin{array}{l}5 \% \\
\text { s.d. }\end{array}$ & alive & $\begin{array}{l}\text { local } \\
\text { rec. }\end{array}$ & $\begin{array}{l}\text { peri- } \\
\text { toneal }\end{array}$ & $\begin{array}{l}\text { liver } \\
\text { rec. }\end{array}$ & $\begin{array}{l}\text { distant } \\
\text { meta. }\end{array}$ & $\mathrm{R}$ & $\begin{array}{l}\text { other } \\
\text { cancer }\end{array}$ & $\begin{array}{l}\text { other } \\
\text { disease }\end{array}$ & $\begin{array}{c}\text { un- } \\
\text { known }\end{array}$ \\
\hline pNO & 71 & 2 & 6 & 63.5 & 41.6 & 28.8 & 24.0 & 17.6 & 9.6 & 11 & 3 & 24 & 10 & 4 & 4 & 0 & 7 & 0 \\
\hline pN1 & 223 & 5 & 20 & 45.9 & 21.8 & 12.2 & 8.3 & 7.2 & 3.8 & 13 & 16 & 85 & 47 & 1 & 19 & 1 & 11 & 5 \\
\hline $\mathrm{pN} 2$ & 352 & 7 & 29 & 42.4 & 21.2 & 11.7 & 8.9 & 6.0 & 2.8 & 15 & 35 & 145 & 53 & 11 & 25 & 4 & 24 & 4 \\
\hline N3 & 262 & 11 & 13 & 45.6 & 19. & 10.8 & 7. & 5. & 2.9 & 13 & 30 & 85 & 35 & 14 & 39 & 1 & 12 & 5 \\
\hline $\mathrm{N} 4$ & 327 & 6 & 28 & 38.7 & 12.6 & 6.7 & 5.9 & 5.1 & 2.7 & 12 & 79 & 91 & 29 & 12 & 42 & 1 & 20 & 7 \\
\hline Not specified & 0 & 0 & 0 & - & - & - & - & - & - & 0 & 0 & 0 & 0 & 0 & 0 & 0 & 0 & 0 \\
\hline$p N 3+p N 4$ & 589 & 17 & 41 & 41.8 & 15.7 & 8.6 & 6.4 & 5.1 & 2.0 & 25 & 109 & 180 & 64 & 26 & 81 & 2 & 32 & 12 \\
\hline
\end{tabular}

Table 32. Resection method of the stomach (resected cases)

\begin{tabular}{|c|c|c|c|c|c|c|c|c|c|c|c|c|c|c|c|c|c|c|}
\hline categories & $\begin{array}{c}\text { total } \\
\text { number }\end{array}$ & $\begin{array}{l}\text { direct } \\
\text { death }\end{array}$ & $\begin{array}{l}\text { lost } \\
\text { f.u. }\end{array}$ & $\begin{array}{l}1 \mathrm{ysr} \\
(\%)\end{array}$ & $\begin{array}{l}2 \text { ysr } \\
(\%)\end{array}$ & $\begin{array}{l}3 \text { ysr } \\
(\%)\end{array}$ & $\begin{array}{l}4 \mathrm{ysr} \\
(\%)\end{array}$ & $\begin{array}{l}5 \mathrm{ysr} \\
(\%)\end{array}$ & $\begin{array}{l}5 \% \\
\text { s.d. }\end{array}$ & alive & $\begin{array}{l}\text { local } \\
\text { rec. }\end{array}$ & $\begin{array}{l}\text { peri- } \\
\text { toneal }\end{array}$ & $\begin{array}{l}\text { liver } \\
\text { rec. }\end{array}$ & $\begin{array}{c}\text { distant } \\
\text { meta. }\end{array}$ & $\mathrm{R}$ & $\begin{array}{l}\text { other } \\
\text { cancer }\end{array}$ & $\begin{array}{l}\text { other } \\
\text { disease }\end{array}$ & $\begin{array}{c}\text { un- } \\
\text { known }\end{array}$ \\
\hline Distal & 5237 & 25 & 346 & 91.3 & 85.3 & 81.2 & 78.7 & 76.8 & 1.2 & 3721 & 155 & 290 & 168 & 47 & 119 & 66 & 223 & 77 \\
\hline Proximal & 242 & 4 & 11 & 88.7 & 81.9 & 75.5 & 72.9 & 69.8 & 6.0 & 160 & 10 & 6 & 7 & 4 & 7 & 9 & 19 & 5 \\
\hline Total & 2439 & 52 & 190 & 75.5 & 61.6 & 55.3 & 51.7 & 49.2 & 2.1 & 1072 & 153 & 441 & 111 & 57 & 140 & 29 & 145 & 49 \\
\hline Not specified & 17 & 0 & 1 & 82.4 & 82.4 & 82.4 & 82.4 & 82.4 & 18.5 & 13 & 0 & 0 & 0 & 0 & 0 & 0 & 3 & 0 \\
\hline
\end{tabular}


Table 33. Resection margins (resected cases)

categories

total direct lost 1 ysr 2 ysr 3 ysr 4 ysr 5 ysr $5 \% \quad$ local peri- liver distant

other other unnumber death f.u. (\%) (\%) (\%) (\%) (\%) s.d. alive rec. toneal rec. meta. $\mathrm{R}$ cancer disease known

$\begin{array}{lllllllllllllllllll}\text { Negative margins } & 7566 & 72 & 514 & 88.5 & 80.4 & 75.8 & 73.0 & 70.8 & 1.1 & 4930 & 270 & 600 & 257 & 96 & 235 & 104 & 361 & 127\end{array}$

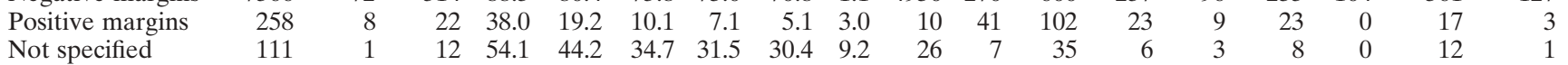

Not specified

Table 34. Lymph node dissection (D) (resected cases)

\begin{tabular}{|c|c|c|c|c|c|c|c|c|c|c|c|c|c|c|c|c|c|c|}
\hline categories & $\begin{array}{c}\text { total } \\
\text { number }\end{array}$ & $\begin{array}{l}\text { direct } \\
\text { death }\end{array}$ & $\begin{array}{l}\text { lost } \\
\text { f.u. }\end{array}$ & $\begin{array}{l}1 \mathrm{ysr} \\
(\%)\end{array}$ & $\begin{array}{l}2 \mathrm{ysr} \\
(\%)\end{array}$ & $\begin{array}{r}3 \text { ysr } \\
(\%)\end{array}$ & $\begin{array}{l}4 \text { ysr } \\
(\%)\end{array}$ & $\begin{array}{l}5 \text { ysr } \\
(\%)\end{array}$ & $\begin{array}{l}5 \% \\
\text { s.d. }\end{array}$ & alive & $\begin{array}{l}\text { local } \\
\text { rec. }\end{array}$ & $\begin{array}{l}\text { peri- } \\
\text { toneal }\end{array}$ & $\begin{array}{l}\text { liver } \\
\text { rec. }\end{array}$ & $\begin{array}{l}\text { distant } \\
\text { meta. }\end{array}$ & $\mathrm{R}$ & $\begin{array}{l}\text { other } \\
\text { cancer }\end{array}$ & $\begin{array}{l}\text { other } \\
\text { disease }\end{array}$ & $\begin{array}{c}\text { un- } \\
\text { known }\end{array}$ \\
\hline D0 & 267 & 3 & 20 & 38.3 & 23.3 & 18.9 & 16.6 & 15.7 & 4.7 & 34 & 25 & 97 & 28 & 6 & 25 & 2 & 24 & 3 \\
\hline D1 & 1518 & 20 & 119 & 81.7 & 74.2 & 69.7 & 67.7 & 65.8 & 2.5 & 902 & 65 & 131 & 65 & 12 & 41 & 36 & 98 & 29 \\
\hline D2 & 5366 & 42 & 357 & 90.8 & 83.4 & 79.0 & 76.2 & 73.9 & 1.2 & 3664 & 156 & 393 & 153 & 68 & 158 & 61 & 230 & 84 \\
\hline D3 & 668 & 11 & 43 & 82.0 & 67.7 & 59.4 & 55.1 & 52.8 & 4.0 & 323 & 58 & 96 & 33 & 20 & 35 & 5 & 30 & 14 \\
\hline D4 & 115 & 5 & 8 & 79.8 & 58.2 & 47.9 & 45.0 & 42.0 & 9.5 & 43 & 14 & 20 & 7 & 2 & 7 & 0 & 8 & 1 \\
\hline Not specified & 1 & 0 & 1 & 100.0 & 0.0 & 0.0 & 0.0 & 0.0 & 0.0 & 0 & 0 & 0 & 0 & 0 & 0 & 0 & 0 & 0 \\
\hline$D 3+D 4$ & 783 & 16 & 51 & 81.7 & 66.3 & 57.7 & 53.6 & 51.2 & 3.7 & 366 & 72 & 116 & 40 & 22 & 42 & 5 & 38 & 15 \\
\hline
\end{tabular}

Table 35. Combined resection of neighboring organs (resected cases)

\begin{tabular}{|c|c|c|c|c|c|c|c|c|c|c|c|c|c|c|c|c|c|c|}
\hline categories & $\begin{array}{c}\text { total } \\
\text { number }\end{array}$ & $\begin{array}{l}\text { direct } \\
\text { death }\end{array}$ & $\begin{array}{l}\text { lost } \\
\text { f.u. }\end{array}$ & $\begin{array}{l}1 \text { ysr } \\
(\%)\end{array}$ & $\begin{array}{l}2 \text { ysr } \\
(\%)\end{array}$ & $\begin{array}{c}3 \text { ysr } \\
(\%)\end{array}$ & $\begin{array}{l}4 \mathrm{ysr} \\
(\%)\end{array}$ & $\begin{array}{l}5 \text { ysr } \\
(\%)\end{array}$ & $\begin{array}{l}5 \% \\
\text { s.d. }\end{array}$ & alive & $\begin{array}{c}\text { local } \\
\text { rec. }\end{array}$ & $\begin{array}{l}\text { peri- } \\
\text { toneal }\end{array}$ & $\begin{array}{l}\text { liver } \\
\text { rec. }\end{array}$ & $\begin{array}{c}\text { distant } \\
\text { meta. }\end{array}$ & $\mathrm{R}$ & $\begin{array}{c}\text { other } \\
\text { cancer }\end{array}$ & $\begin{array}{c}\text { other } \\
\text { disease }\end{array}$ & $\begin{array}{c}\text { un- } \\
\text { known }\end{array}$ \\
\hline No comb. resection & 5530 & 33 & 373 & 90.8 & 84.8 & 80.7 & 78.3 & 76.3 & 1.2 & 3899 & 172 & 318 & 161 & 53 & 147 & 60 & 241 & 73 \\
\hline Comb. resection & 2394 & 48 & 175 & 76.2 & 62.0 & 55.7 & 52.0 & 49.3 & 2.1 & 1061 & 146 & 419 & 122 & 55 & 119 & 44 & 147 & 58 \\
\hline Not specified & 11 & 0 & 0 & 81.8 & 72.7 & 63.6 & 63.6 & 54.5 & 30.0 & 6 & 0 & 0 & 3 & 0 & 0 & 0 & 2 & 0 \\
\hline
\end{tabular}

Table 36. Resected organs, combined (resected cases)

\begin{tabular}{|c|c|c|c|c|c|c|c|c|c|c|c|c|c|c|c|c|c|c|}
\hline categories & $\begin{array}{c}\text { total } \\
\text { number }\end{array}$ & $\begin{array}{l}\text { direct } \\
\text { death }\end{array}$ & $\begin{array}{l}\text { lost } \\
\text { f.u. }\end{array}$ & $\begin{array}{l}1 \text { ysr } \\
(\%)\end{array}$ & $\begin{array}{l}2 \mathrm{ysr} \\
(\%)\end{array}$ & $\begin{array}{r}3 \text { ysr } \\
(\%)\end{array}$ & $\begin{array}{l}4 \mathrm{ysr} \\
(\%)\end{array}$ & $\begin{array}{l}5 \mathrm{ysr} \\
(\%)\end{array}$ & $\begin{array}{l}5 \% \\
\text { s.d. }\end{array}$ & alive & $\begin{array}{l}\text { local } \\
\text { rec. }\end{array}$ & $\begin{array}{l}\text { peri- } \\
\text { toneal }\end{array}$ & $\begin{array}{l}\text { liver } \\
\text { rec. }\end{array}$ & $\begin{array}{l}\text { distant } \\
\text { meta. }\end{array}$ & $\mathrm{R}$ & $\begin{array}{l}\text { other } \\
\text { cancer }\end{array}$ & $\begin{array}{c}\text { other } \\
\text { disease }\end{array}$ & $\begin{array}{c}\text { un- } \\
\text { known }\end{array}$ \\
\hline Spleen & 1515 & 34 & 111 & 75.3 & 58.9 & 52.6 & 48.4 & 46.0 & 2.6 & 622 & 102 & 295 & 71 & 40 & 94 & 16 & 90 & 40 \\
\hline Pancreas & 726 & 18 & 49 & 72.6 & 53.4 & 46.9 & 42.1 & 39.0 & 3.7 & 253 & 55 & 158 & 39 & 25 & 62 & 6 & 41 & 20 \\
\hline Colon & 171 & 5 & 12 & 59.2 & 43.3 & 34.4 & 31.1 & 27.1 & 7.1 & 41 & 8 & 59 & 10 & 4 & 13 & 3 & 15 & 1 \\
\hline Adrenal gland & 76 & 1 & 3 & 81.6 & 72.3 & 62.9 & 57.5 & 50.4 & 11.6 & 36 & 5 & 16 & 4 & 2 & 3 & 1 & 3 & 2 \\
\hline Liver & 101 & 3 & 9 & 60.0 & 47.8 & 44.4 & 41.0 & 33.0 & 9.8 & 29 & 8 & 13 & 19 & 1 & 8 & 2 & 6 & 3 \\
\hline Gall bladder & 730 & 9 & 58 & 79.5 & 69.4 & 63.8 & 60.9 & 58.3 & 3.8 & 383 & 43 & 105 & 41 & 5 & 13 & 16 & 40 & 17 \\
\hline Diaphragma & 27 & 1 & 1 & 70.4 & 44.4 & 33.3 & 21.6 & 17.3 & 15.0 & 4 & 2 & 5 & 3 & 2 & 6 & 0 & 2 & 1 \\
\hline
\end{tabular}

Table 37. Curative potential of resection (resected cases)

\begin{tabular}{|c|c|c|c|c|c|c|c|c|c|c|c|c|c|c|c|c|c|c|}
\hline categories & $\begin{array}{c}\text { total } \\
\text { number }\end{array}$ & $\begin{array}{l}\text { direct } \\
\text { death }\end{array}$ & $\begin{array}{l}\text { lost } \\
\text { f.u. }\end{array}$ & $\begin{array}{l}1 \mathrm{ysr} \\
(\%)\end{array}$ & $\begin{array}{l}2 \text { ysr } \\
(\%)\end{array}$ & $\begin{array}{r}3 \text { ysr } \\
(\%)\end{array}$ & $\begin{array}{l}4 \text { ysr } \\
(\%)\end{array}$ & $\begin{array}{l}5 \text { ysr } \\
(\%)\end{array}$ & $\begin{array}{l}5 \% \\
\text { s.d. }\end{array}$ & alive & $\begin{array}{l}\text { local } \\
\text { rec. }\end{array}$ & $\begin{array}{l}\text { peri- } \\
\text { toneal }\end{array}$ & $\begin{array}{l}\text { liver } \\
\text { rec. }\end{array}$ & $\begin{array}{c}\text { distant } \\
\text { meta. }\end{array}$ & $\mathrm{R}$ & $\begin{array}{l}\text { other } \\
\text { cancer }\end{array}$ & $\begin{array}{c}\text { other } \\
\text { disease }\end{array}$ & $\begin{array}{c}\text { un- } \\
\text { known }\end{array}$ \\
\hline & & & & & & & & & & 4105 & & & & & & & & 69 \\
\hline & & 2 & 146 & 85.5 & 70.3 & 61 & 55.1 & 51.4 & 2.5 & 796 & 123 & 252 & 70 & 5 & 104 & 22 & 107 & 41 \\
\hline Resection & 1235 & 31 & 96 & 44.0 & 19.9 & 11.3 & 8.5 & 6.5 & 1.5 & 64 & 163 & 434 & 174 & 42 & 129 & 7 & 74 & 21 \\
\hline Not specified & 2 & 0 & 1 & 100.0 & 100.0 & 100.0 & 100.0 & 100.0 & 0.0 & 1 & 0 & 0 & 0 & 0 & 0 & 0 & 0 & 0 \\
\hline Resection $A+B$ & 6698 & 50 & 451 & 94.1 & 88.3 & 84.1 & 81.3 & 79.0 & 1.0 & 4901 & 155 & 303 & 112 & 66 & 137 & 97 & 316 & 110 \\
\hline
\end{tabular}

Footnote for Table 33: Resection margins

Negative resection margins means no cancer involvement of either proximal or distal margins. Positive resection margin means cancer involvement of the proximal or distal resection margin or both.
Footnote for Tables 35, 36: Combined resection of neighboring organs

The organs are the spleen, liver, pancreas, transverse colon, transverse mesocolon, gallbladder, adrenal gland, ovary, etc. Resection of the greater or lesser omentum, the anterior sheet of the transverse mesocolon, the abdominal esophagus and the first portion of the duodenum are not included in this category. 


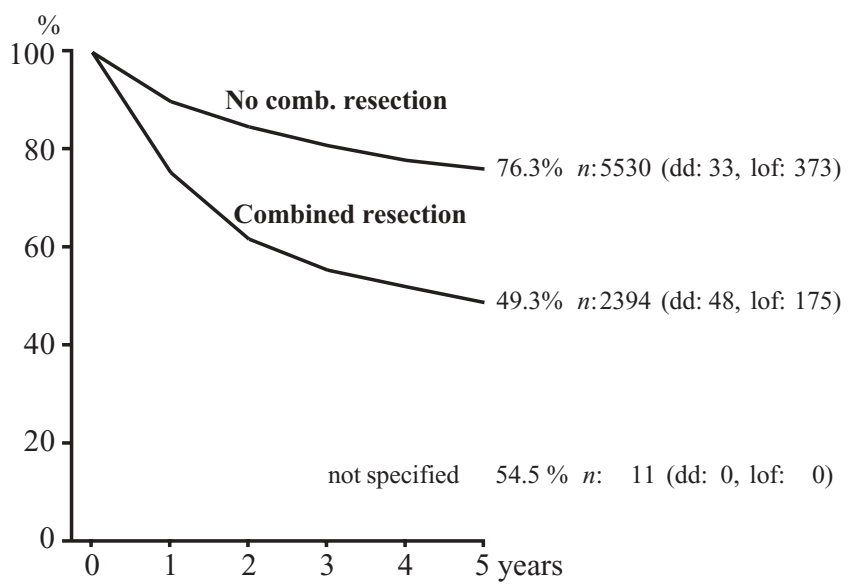

Fig. 6. Combined resection of neighboring organs

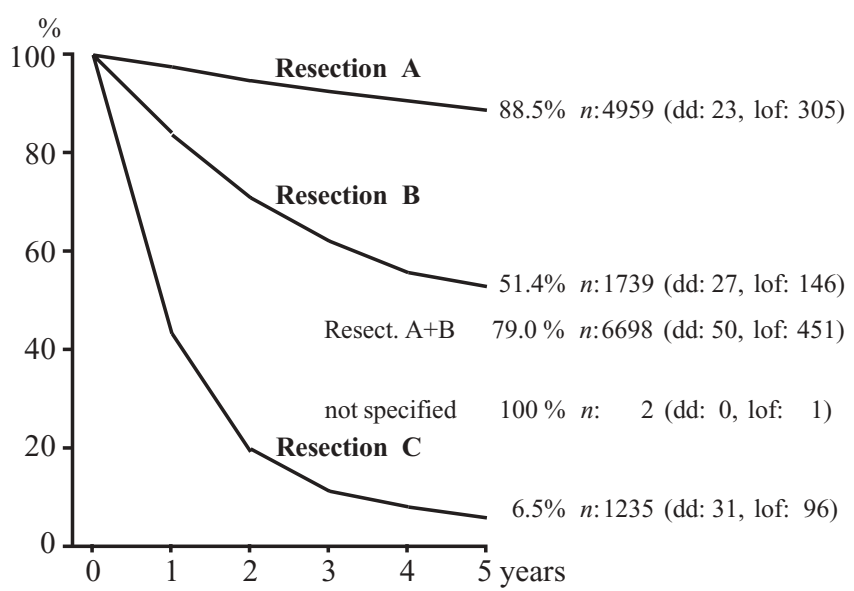

Fig. 7. Curative potential of resection

Table 38. Japanese stage (resected cases)

\begin{tabular}{|c|c|c|c|c|c|c|c|c|c|c|c|c|c|c|c|c|c|c|}
\hline categories & $\begin{array}{c}\text { total } \\
\text { number }\end{array}$ & $\begin{array}{l}\text { direct } \\
\text { death }\end{array}$ & $\begin{array}{l}\text { lost } \\
\text { f.u. }\end{array}$ & $\begin{array}{l}1 \mathrm{ysr} \\
(\%)\end{array}$ & $\begin{array}{l}2 \mathrm{ysr} \\
(\%)\end{array}$ & $\begin{array}{c}3 \text { ysr } \\
(\%)\end{array}$ & $\begin{array}{l}4 \text { ysr } \\
(\%)\end{array}$ & $\begin{array}{l}5 \text { ysr } \\
(\%)\end{array}$ & $\begin{array}{l}5 \% \\
\text { s.d. }\end{array}$ & alive & $\begin{array}{c}\text { local } \\
\text { rec. }\end{array}$ & $\begin{array}{l}\text { peri- } \\
\text { toneal }\end{array}$ & $\begin{array}{c}\text { liver } \\
\text { rec. }\end{array}$ & $\begin{array}{c}\text { distant } \\
\text { meta. }\end{array}$ & $\mathrm{R}$ & $\begin{array}{l}\text { other } \\
\text { cancer }\end{array}$ & $\begin{array}{c}\text { other } \\
\text { disease }\end{array}$ & $\begin{array}{c}\text { un- } \\
\text { known }\end{array}$ \\
\hline Stage I & 4614 & 21 & 282 & 97.1 & 95.2 & 93.0 & 91.5 & 89.9 & 0.9 & 3884 & 22 & 36 & 24 & 8 & 22 & 68 & 192 & 55 \\
\hline Stage II & 834 & 10 & 61 & 93.9 & 84.1 & 77.9 & 73.5 & 69.1 & 3.3 & 528 & 26 & 48 & 28 & 15 & 26 & 13 & 54 & 25 \\
\hline Stage III & 1285 & 23 & 120 & 81.5 & 63.1 & 51.7 & 45.0 & 41.5 & 2.9 & 463 & 120 & 231 & 58 & 48 & 95 & 20 & 76 & 31 \\
\hline Stage IV & 1200 & 27 & 84 & 44.4 & 20.8 & 13.2 & 10.5 & 9.0 & 1.8 & 90 & 150 & 422 & 176 & 37 & 123 & 3 & 68 & 20 \\
\hline Not specified & 2 & 0 & 1 & 100.0 & 100.0 & 100.0 & 100.0 & 100.0 & 0.0 & 1 & 0 & 0 & 0 & 0 & 0 & 0 & 0 & 0 \\
\hline
\end{tabular}

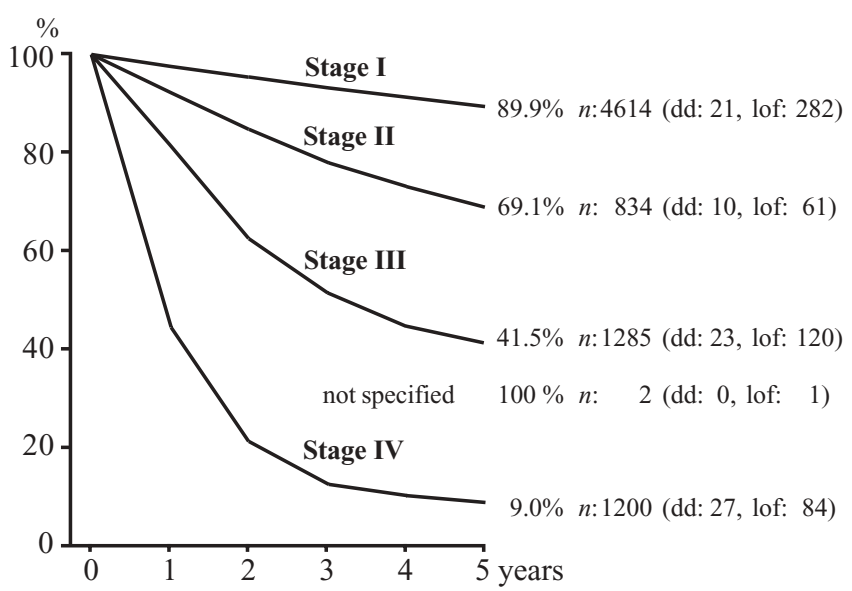

Fig. 8. Japanese stage

Footnote for Tables 38-41:

Japanese staging in the 12th Japanese edition [5]

Japanese staging, 11th ed., was not correlated with survivals. To remove the contradiction, Japanese staging, 12th ed., was published in 1993, and it was principally the same as UICC-TNM staging, 5th ed. For the continuity of statistics, this analysis used the Japanese staging, 12 th ed. As the staging was evaluated by a combination of T, N, and $\mathrm{M}$, it was possible to recalculate the Japanese staging, 12th ed., from the collected data (see table at right). The UICC-TNM staging, 5th ed., could also be recalculated.

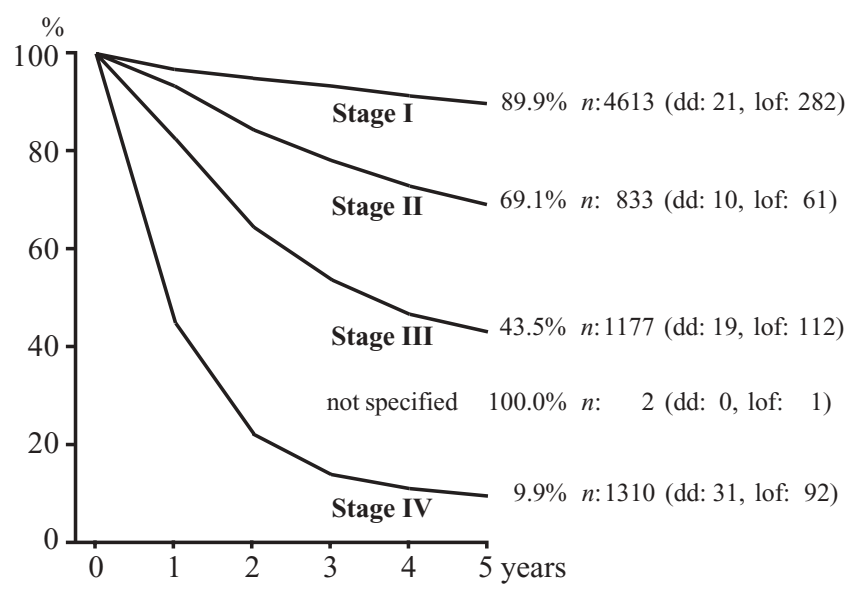

Fig. 9. UICC TNM stage, 5th ed.

\begin{tabular}{|c|c|c|c|c|c|c|}
\hline & No & N1 & $\mathrm{N} 2$ & $\mathrm{~N} 3$ & $\begin{array}{l}\mathrm{P} 0, \mathrm{H} 1, \\
\quad<\mathrm{N} 2\end{array}$ & $\mathrm{~N} 3 / \mathrm{N} 4$ \\
\hline $\mathrm{T} 1$ & IA & IB & II & IIIA & \multirow{3}{*}{ IVA } & \\
\hline $\mathrm{T} 2$ & IB & II & IIIA & IIIB & & \\
\hline $\mathrm{T} 3$ & II & IIIA & IIIB & IVA & & \\
\hline $\mathrm{T} 4$ & IIIA & IIIB & & \multirow{3}{*}{\multicolumn{3}{|c|}{$\begin{array}{c}+\mathrm{P} 1 \text { or } \mathrm{H} 1 \text { or } \mathrm{M} 1 \\
\text { IVB }\end{array}$}} \\
\hline $\mathrm{P} 1, \mathrm{H} 0,<\mathrm{T} 3$ & \multicolumn{3}{|c|}{ IVA } & & & \\
\hline $\mathrm{T} 4$ & & & & & & \\
\hline
\end{tabular}


Table 39. Japanese stage (resected cases)

\begin{tabular}{|c|c|c|c|c|c|c|c|c|c|c|c|c|c|c|c|c|c|c|}
\hline categories & $\begin{array}{c}\text { total } \\
\text { number }\end{array}$ & $\begin{array}{l}\text { direct } \\
\text { death }\end{array}$ & $\begin{array}{l}\text { lost } \\
\text { f.u. }\end{array}$ & $\begin{array}{l}1 \mathrm{ysr} \\
(\%)\end{array}$ & $\begin{array}{l}2 \mathrm{ysr} \\
(\%)\end{array}$ & $\begin{array}{l}3 \text { ysr } \\
(\%)\end{array}$ & $\begin{array}{l}4 \mathrm{ysr} \\
(\%)\end{array}$ & $\begin{array}{l}5 \mathrm{ysr} \\
(\%)\end{array}$ & $\begin{array}{l}5 \% \\
\text { s.d. }\end{array}$ & alive & $\begin{array}{l}\text { local } \\
\text { rec. }\end{array}$ & $\begin{array}{l}\text { peri- } \\
\text { toneal }\end{array}$ & $\begin{array}{l}\text { liver } \\
\text { rec. }\end{array}$ & $\begin{array}{c}\text { distant } \\
\text { meta. }\end{array}$ & $\mathrm{R}$ & $\begin{array}{l}\text { other } \\
\text { cancer }\end{array}$ & $\begin{array}{c}\text { other } \\
\text { disease }\end{array}$ & $\begin{array}{c}\text { un- } \\
\text { known }\end{array}$ \\
\hline Stage IA & 3505 & 12 & 211 & 97.5 & 96.0 & 94.3 & 93.0 & 91.6 & 1.0 & 3010 & 9 & 11 & 5 & 1 & 10 & 55 & 147 & 34 \\
\hline Stage IB & 1109 & 9 & 71 & 96.0 & 92.5 & 89.1 & 86.8 & 84.7 & 2.2 & 874 & 13 & 25 & 19 & 7 & 12 & 13 & 45 & 21 \\
\hline Stage II & 834 & 10 & 61 & 93.9 & 84.1 & 77.9 & 73.5 & 69.1 & 3.3 & 528 & 26 & 48 & 28 & 15 & 26 & 13 & 54 & 25 \\
\hline Stage IIIA & 782 & 13 & 77 & 86.7 & 71.2 & 59.6 & 53.3 & 50.1 & 3.7 & 342 & 54 & 115 & 40 & 23 & 43 & 13 & 44 & 18 \\
\hline Stage IIIB & 503 & 10 & 43 & 73.5 & 50.5 & 39.4 & 32.0 & 28.1 & 4.2 & 121 & 66 & 116 & 18 & 25 & 52 & 7 & 32 & 13 \\
\hline Stage IVA & 324 & 7 & 21 & 61.3 & 35.0 & 25.2 & 19.8 & 16.6 & 4.3 & 48 & 38 & 96 & 42 & 8 & 34 & 2 & 24 & 4 \\
\hline Stage IVB & 876 & 20 & 63 & 38.0 & 15.5 & 8.6 & 7.0 & 6.1 & 1.7 & 42 & 112 & 326 & 134 & 29 & 89 & 1 & 44 & 16 \\
\hline Not specified & 2 & 0 & 1 & 100.0 & 100.0 & 100.0 & 100.0 & 100.0 & 0.0 & 1 & 0 & 0 & 0 & 0 & 0 & 0 & 0 & 0 \\
\hline
\end{tabular}

Table 40. Japanese stage by D0 or D1 dissection (resected cases)

\begin{tabular}{|c|c|c|c|c|c|c|c|c|c|c|c|c|c|c|c|c|c|c|}
\hline categories & $\begin{array}{c}\text { total } \\
\text { number }\end{array}$ & $\begin{array}{l}\text { direct } \\
\text { death }\end{array}$ & $\begin{array}{l}\text { lost } \\
\text { f.u. }\end{array}$ & $\begin{array}{l}1 \mathrm{ysr} \\
(\%)\end{array}$ & $\begin{array}{l}2 \mathrm{ysr} \\
(\%)\end{array}$ & $\begin{array}{c}3 \text { ysr } \\
(\%)\end{array}$ & $\begin{array}{l}4 y s r \\
(\%)\end{array}$ & $\begin{array}{l}5 \text { ysr } \\
(\%)\end{array}$ & $\begin{array}{l}5 \% \\
\text { s.d. }\end{array}$ & alive & $\begin{array}{l}\text { local } \\
\text { rec. }\end{array}$ & $\begin{array}{l}\text { peri- } \\
\text { toneal }\end{array}$ & $\begin{array}{l}\text { liver } \\
\text { rec. }\end{array}$ & $\begin{array}{c}\text { distant } \\
\text { meta. }\end{array}$ & $\mathrm{R}$ & $\begin{array}{l}\text { other } \\
\text { cancer }\end{array}$ & $\begin{array}{c}\text { other } \\
\text { disease }\end{array}$ & $\begin{array}{c}\text { un- } \\
\text { known }\end{array}$ \\
\hline Stage I & 1101 & 8 & 74 & 95.5 & 92.9 & 89.8 & 88.0 & 85.8 & 2.1 & 877 & 7 & 6 & 4 & 1 & 3 & 30 & 72 & 19 \\
\hline Stage II & 68 & 2 & 3 & 80.5 & 61.9 & 52.6 & 48.0 & 46.4 & 12.4 & 30 & 6 & 3 & 3 & 2 & 3 & 1 & 11 & 4 \\
\hline Stage III & 88 & 1 & 13 & 65.7 & 40.7 & 30.7 & 26.3 & 21.8 & 9.7 & 14 & 15 & 15 & 4 & 2 & 8 & 5 & 10 & 1 \\
\hline Stage IV & 527 & 12 & 49 & 32.5 & 13.3 & 6.5 & 4.5 & 3.9 & 1.9 & 14 & 62 & 204 & 82 & 13 & 52 & 2 & 29 & 8 \\
\hline Not specified & 1 & 0 & 0 & 100.0 & 100.0 & 100.0 & 100.0 & 100.0 & 0.0 & 1 & 0 & 0 & 0 & 0 & 0 & 0 & 0 & 0 \\
\hline
\end{tabular}

Table 41. Japanese stage by D2, D3, or D4 dissection (resected cases)

\begin{tabular}{|c|c|c|c|c|c|c|c|c|c|c|c|c|c|c|c|c|c|c|}
\hline categories & $\begin{array}{c}\text { total } \\
\text { number }\end{array}$ & $\begin{array}{l}\text { direct } \\
\text { death }\end{array}$ & $\begin{array}{l}\text { lost } \\
\text { f.u. }\end{array}$ & $\begin{array}{l}1 \text { ysr } \\
(\%)\end{array}$ & $\begin{array}{l}2 \text { ysr } \\
(\%)\end{array}$ & $\begin{array}{r}3 \text { ysr } \\
(\%)\end{array}$ & $\begin{array}{l}4 \text { ysr } \\
(\%)\end{array}$ & $\begin{array}{l}5 \text { ysr } \\
(\%)\end{array}$ & $\begin{array}{l}5 \% \\
\text { s.d. }\end{array}$ & alive & $\begin{array}{l}\text { local } \\
\text { rec. }\end{array}$ & $\begin{array}{l}\text { peri- } \\
\text { toneal }\end{array}$ & $\begin{array}{l}\text { liver } \\
\text { rec. }\end{array}$ & $\begin{array}{c}\text { distant } \\
\text { meta. }\end{array}$ & $\mathrm{R}$ & $\begin{array}{l}\text { other } \\
\text { cancer }\end{array}$ & $\begin{array}{c}\text { other } \\
\text { disease }\end{array}$ & $\begin{array}{c}\text { un- } \\
\text { known }\end{array}$ \\
\hline Stage I & 3513 & 13 & 208 & 97.6 & 95.9 & 94.0 & 92.6 & 91.2 & 1.0 & 3007 & 15 & 30 & 20 & 7 & 19 & 38 & 120 & 36 \\
\hline Stage II & 766 & 8 & 58 & 95.1 & 86.1 & 80.2 & 75.8 & 71.1 & 3.4 & 498 & 20 & 45 & 25 & 13 & 23 & 12 & 43 & 21 \\
\hline Stage III & 1197 & 22 & 107 & 82.7 & 64.7 & 53.2 & 46.3 & 42.8 & 3.0 & 449 & 105 & 216 & 54 & 46 & 87 & 15 & 66 & 30 \\
\hline Stage IV & 673 & 15 & 35 & 53.4 & 26.5 & 18.1 & 14.8 & 12.7 & 2.7 & 76 & 88 & 218 & 94 & 24 & 71 & 1 & 39 & 12 \\
\hline Not specified & 0 & 0 & 0 & - & - & - & - & - & - & 0 & 0 & 0 & 0 & 0 & 0 & 0 & 0 & 0 \\
\hline
\end{tabular}

Table 42. UICC TNM stage, 5th ed. (resected cases)

\begin{tabular}{|c|c|c|c|c|c|c|c|c|c|c|c|c|c|c|c|c|c|c|}
\hline categories & $\begin{array}{c}\text { total } \\
\text { number }\end{array}$ & $\begin{array}{l}\text { direct } \\
\text { death }\end{array}$ & $\begin{array}{l}\text { lost } \\
\text { f.u. }\end{array}$ & $\begin{array}{l}1 \mathrm{ysr} \\
(\%)\end{array}$ & $\begin{array}{l}2 \mathrm{ysr} \\
(\%)\end{array}$ & $\begin{array}{r}3 \text { ysr } \\
(\%)\end{array}$ & $\begin{array}{l}4 \text { ysr } \\
(\%)\end{array}$ & $\begin{array}{l}5 \text { ysr } \\
(\%)\end{array}$ & $\begin{array}{l}5 \% \\
\text { s.d. }\end{array}$ & alive & $\begin{array}{l}\text { local } \\
\text { rec. }\end{array}$ & $\begin{array}{l}\text { peri- } \\
\text { toneal }\end{array}$ & $\begin{array}{l}\text { liver } \\
\text { rec. }\end{array}$ & $\begin{array}{l}\text { distant } \\
\text { meta. }\end{array}$ & $\mathrm{R}$ & $\begin{array}{l}\text { other } \\
\text { cancer }\end{array}$ & $\begin{array}{c}\text { other } \\
\text { disease }\end{array}$ & $\begin{array}{c}\text { un- } \\
\text { known }\end{array}$ \\
\hline Stage I & 4613 & 21 & 282 & 97.1 & 95.2 & 93.1 & 91.5 & 89.9 & 0.9 & 3884 & 22 & 36 & 24 & 8 & 22 & 68 & 191 & 55 \\
\hline Stage II & 833 & 10 & 61 & 93.9 & 84.3 & 78.0 & 73.6 & 69.1 & 3.3 & 528 & 26 & 48 & 27 & 15 & 26 & 13 & 54 & 25 \\
\hline Stage III & 1177 & 19 & 112 & 82.7 & 64.8 & 53.7 & 46.9 & 43.5 & 3.0 & 444 & 104 & 206 & 55 & 36 & 82 & 19 & 73 & 27 \\
\hline Stage IV & 1310 & 31 & 92 & 46.4 & 22.7 & 14.6 & 11.6 & 9.9 & 1.7 & 109 & 166 & 447 & 180 & 49 & 136 & 4 & 72 & 24 \\
\hline Not specified & 2 & 0 & 1 & 100.0 & 100.0 & 100.0 & 100.0 & 100.0 & 0.0 & 1 & 0 & 0 & 0 & 0 & 0 & 0 & 0 & 0 \\
\hline
\end{tabular}

Table 43. UICC TNM stage, 5th ed. (resected cases)

\begin{tabular}{|c|c|c|c|c|c|c|c|c|c|c|c|c|c|c|c|c|c|c|}
\hline categories & $\begin{array}{c}\text { total } \\
\text { number }\end{array}$ & $\begin{array}{l}\text { direct } \\
\text { death }\end{array}$ & $\begin{array}{l}\text { lost } \\
\text { f.u. }\end{array}$ & $\begin{array}{l}1 \mathrm{ysr} \\
(\%)\end{array}$ & $\begin{array}{l}2 y s r \\
(\%)\end{array}$ & $\begin{array}{l}3 \text { ysr } \\
(\%)\end{array}$ & $\begin{array}{l}4 y s r \\
(\%)\end{array}$ & $\begin{array}{l}5 \text { ysr } \\
(\%)\end{array}$ & $\begin{array}{l}5 \% \\
\text { s.d. }\end{array}$ & alive & $\begin{array}{l}\text { local } \\
\text { rec. }\end{array}$ & $\begin{array}{l}\text { peri- } \\
\text { toneal }\end{array}$ & $\begin{array}{c}\text { liver } \\
\text { rec. }\end{array}$ & $\begin{array}{c}\text { distant } \\
\text { meta. }\end{array}$ & $\mathrm{R}$ & $\begin{array}{l}\text { other } \\
\text { cancer }\end{array}$ & $\begin{array}{c}\text { other } \\
\text { disease }\end{array}$ & $\begin{array}{c}\text { un- } \\
\text { known }\end{array}$ \\
\hline Stage Ia & 3505 & 12 & 211 & 97.5 & 96.0 & 94.3 & 93.0 & 91.6 & 1.0 & 3010 & 9 & 11 & 5 & 1 & 10 & 55 & 147 & 34 \\
\hline Stage Ib & 1108 & 9 & 71 & 96.1 & 92.5 & 89.2 & 86.9 & 84.7 & 2.2 & 874 & 13 & 25 & 19 & 7 & 12 & 13 & 44 & 21 \\
\hline Stage II & 833 & 10 & 61 & 93.9 & 84.3 & 78.0 & 73.6 & 69.1 & 3.3 & 528 & 26 & 48 & 27 & 15 & 26 & 13 & 54 & 25 \\
\hline Stage IIIa & 764 & 12 & 76 & 87.1 & 71.8 & 60.1 & 53.8 & 50.5 & 3.8 & 337 & 51 & 111 & 40 & 22 & 42 & 12 & 43 & 18 \\
\hline Stage IIIb & 413 & 7 & 36 & 74.5 & 52.1 & 41.8 & 34.2 & 30.5 & 4.7 & 107 & 53 & 95 & 15 & 14 & 40 & 4 & 30 & 9 \\
\hline Stage IV & 1310 & 31 & 92 & 46.4 & 22.7 & 14.6 & 11.6 & 9.9 & 1.7 & 109 & 166 & 447 & 180 & 49 & 136 & 4 & 72 & 24 \\
\hline Not specified & 2 & 0 & 1 & 100.0 & 100.0 & 100.0 & 100.0 & 100.0 & 0.0 & 1 & 0 & 0 & 0 & 0 & 0 & 0 & 0 & 0 \\
\hline
\end{tabular}


Table 44. UICC TNM stage, 5th ed. by D0 or D1 dissection (resected cases)

\begin{tabular}{|c|c|c|c|c|c|c|c|c|c|c|c|c|c|c|c|c|c|c|}
\hline categories & $\begin{array}{c}\text { total } \\
\text { number }\end{array}$ & $\begin{array}{l}\text { direct } \\
\text { death }\end{array}$ & $\begin{array}{l}\text { lost } \\
\text { f.u. }\end{array}$ & $\begin{array}{l}1 \mathrm{ysr} \\
(\%)\end{array}$ & $\begin{array}{l}2 \text { ysr } \\
(\%)\end{array}$ & $\begin{array}{r}3 \text { ysr } \\
(\%)\end{array}$ & $\begin{array}{l}4 \text { ysr } \\
(\%)\end{array}$ & $\begin{array}{l}5 y s r \\
(\%)\end{array}$ & $\begin{array}{l}5 \% \\
\text { s.d. }\end{array}$ & alive & $\begin{array}{l}\text { local } \\
\text { rec. }\end{array}$ & $\begin{array}{l}\text { peri- } \\
\text { toneal }\end{array}$ & $\begin{array}{l}\text { liver } \\
\text { rec. }\end{array}$ & $\begin{array}{l}\text { distant } \\
\text { meta. }\end{array}$ & $\mathrm{R}$ & $\begin{array}{l}\text { other } \\
\text { cancer }\end{array}$ & $\begin{array}{l}\text { other } \\
\text { disease }\end{array}$ & $\begin{array}{c}\text { un- } \\
\text { known }\end{array}$ \\
\hline Stage I & 1100 & 8 & 74 & 95.6 & 92.9 & 89.9 & 88.1 & 85.9 & 2.1 & 877 & 7 & 6 & 4 & 1 & 3 & 30 & 71 & 19 \\
\hline Stage II & 68 & 2 & 3 & 80.5 & 61.9 & 52.6 & 48.0 & 46.4 & 12.4 & 30 & 6 & 3 & 3 & 2 & 3 & 1 & 11 & 4 \\
\hline Stage III & 76 & 1 & 13 & 68.3 & 45.0 & 34.8 & 29.6 & 24.2 & 11.0 & 13 & 10 & 11 & 4 & 2 & 8 & 4 & 9 & 1 \\
\hline Stage IV & 540 & 12 & 49 & 32.8 & 13.3 & 6.5 & 4.6 & 4.0 & 1.9 & 15 & 67 & 208 & 82 & 13 & 52 & 3 & 31 & 8 \\
\hline Not specified & 1 & 0 & 0 & 100.0 & 100.0 & 100.0 & 100.0 & 100.0 & 0.0 & 1 & 0 & 0 & 0 & 0 & 0 & 0 & 0 & 0 \\
\hline
\end{tabular}

Table 45. UICC TNM stage, 5th ed. by D2, D3, or D4 dissection (resected cases)

\begin{tabular}{|c|c|c|c|c|c|c|c|c|c|c|c|c|c|c|c|c|c|c|}
\hline categories & $\begin{array}{l}\text { total } \\
\text { number }\end{array}$ & $\begin{array}{l}\text { direct } \\
\text { death }\end{array}$ & $\begin{array}{l}\text { lost } \\
\text { f.u. }\end{array}$ & $\begin{array}{l}1 \mathrm{ysr} \\
(\%)\end{array}$ & $\begin{array}{l}2 \text { ysr } \\
(\%)\end{array}$ & $\begin{array}{r}3 \text { ysr } \\
(\%)\end{array}$ & $\begin{array}{l}4 \text { ysr } \\
(\%)\end{array}$ & $\begin{array}{l}5 \mathrm{ysr} \\
(\%)\end{array}$ & $\begin{array}{l}5 \% \\
\text { s.d. }\end{array}$ & alive & $\begin{array}{l}\text { local } \\
\text { rec. }\end{array}$ & $\begin{array}{l}\text { peri- } \\
\text { toneal }\end{array}$ & $\begin{array}{l}\text { liver } \\
\text { rec. }\end{array}$ & $\begin{array}{l}\text { distant } \\
\text { meta. }\end{array}$ & $\mathrm{R}$ & $\begin{array}{l}\text { other } \\
\text { cancer }\end{array}$ & $\begin{array}{l}\text { other } \\
\text { disease }\end{array}$ & $\begin{array}{c}\text { un- } \\
\text { known }\end{array}$ \\
\hline Stage I & 3513 & 13 & 208 & 97.6 & 95.9 & 94.0 & 92.6 & 91.2 & 1.0 & 3007 & 15 & 30 & 20 & 7 & 19 & 38 & 120 & 36 \\
\hline Stage II & 765 & 8 & 58 & 95.1 & 86.2 & 80.3 & 75.9 & 71.1 & 3.4 & 498 & 20 & 45 & 24 & 13 & 23 & 12 & 43 & 21 \\
\hline Stage III & 1101 & 18 & 99 & 83.6 & 66.1 & 54.9 & 48.0 & 44.7 & 3.1 & 431 & 94 & 195 & 51 & 34 & 74 & 15 & 64 & 26 \\
\hline Stage IV & 770 & 19 & 43 & 55.8 & 29.1 & 19.9 & 16.2 & 13.7 & 2.6 & 94 & 99 & 239 & 98 & 36 & 84 & 1 & 41 & 16 \\
\hline Not specifieds & 0 & 0 & 0 & - & - & - & - & - & - & 0 & 0 & 0 & 0 & 0 & 0 & 0 & 0 & 0 \\
\hline
\end{tabular}

